\title{
Universiteit
}

Leiden

The Netherlands

\section{Packing geometry and statistics of force networks in granular media}

Snoeijer, J.H.; Hecke, M.L. van; Somfai, E.; Saarloos, W. van

\section{Citation}

Snoeijer, J. H., Hecke, M. L. van, Somfai, E., \& Saarloos, W. van. (2004). Packing geometry and statistics of force networks in granular media. Physical Review E, 70(1), 011301. doi:10.1103/PhysRevE.70.011301

Version: $\quad$ Not Applicable (or Unknown)

License: $\quad$ Leiden University Non-exclusive license

Downloaded from: https://hdl.handle.net/1887/66540

Note: To cite this publication please use the final published version (if applicable). 


\title{
Packing geometry and statistics of force networks in granular media
}

\author{
Jacco H. Snoeijer, ${ }^{1}$ Martin van Hecke, ${ }^{2}$ Ellák Somfai, ${ }^{1}$ and Wim van Saarloos ${ }^{1}$ \\ ${ }^{1}$ Instituut-Lorentz, Universiteit Leiden, Postbus 9506, 2300 RA Leiden, The Netherlands \\ ${ }^{2}$ Kamerlingh Onnes Lab, Universiteit Leiden, Postbus 9504, 2300 RA Leiden, The Netherlands
}

(Received 9 October 2003; published 8 July 2004)

\begin{abstract}
The relation between packing geometry and force network statistics is studied for granular media. Based on simulations of two-dimensional packings of Hertzian spheres, we develop a geometrical framework relating the distribution of interparticle forces $P(f)$ to the weight distribution $\mathcal{P}(w)$, which is measured in experiments. We apply this framework to reinterpret recent experimental data on strongly deformed packings and suggest that the observed changes of $\mathcal{P}(w)$ are dominated by changes in contact network while $P(f)$ remains relatively unaltered. We furthermore investigate the role of packing disorder in the context of the $q$ model and address the question of how force fluctuations build up as a function of the distance beneath the top surface.
\end{abstract}

DOI: 10.1103/PhysRevE.70.011301

PACS number(s): 45.70.Cc, 46.65.+g, 05.40.-a

\section{INTRODUCTION}

Inside a granular material forces are distributed very inhomogeneously: a small number of particles carries a large fraction of the internal forces [1]. These large fluctuations are reflected in the force probability density functions, which typically decay exponentially [2-5]. The behavior for small forces is not as well understood as the generic exponential tail: the $q$ model appears to predict a vanishing probability density for small forces [5], whereas experiments and simulations clearly show that this probability remains nonzero [2-4]. The characterization and understanding of this probability remains a challenge, especially since the force distribution is believed to play an important role for the dynamical arrest or "jamming" of granular and other disordered materials [6]. In particular, the force distribution has been observed to develop a small peak (around the average value) in simulations of supercooled liquids, foams, and granular matter undergoing a jamming transition [6,7]. However, there is still no microscopic understanding how this effect relates to the properties of the force network.

This paper is a full exposition and expansion of an approach which was briefly outlined in [8]. We will unravel the effect of the local contact geometry on the distributions of interparticle force $F$ and effective particle weight $W$; the weight is defined as the sum of the vertical components of all downward pointing forces on a particle-see Fig. 1. While the distribution of forces $F$ is the primary object one ultimately wishes to characterize, it is difficult to access experimentally. Experiments with photoelastic materials are able to depict the spatial structure of bulk forces in two dimensions (2D), but their precision to resolve individual contact forces is limited [9]. Only recently, there have been first reports of 3D bulk measurements on forces in compressed emulsions [10]. Most quantitative information on the force probability distribution is at present only accessible through measurements of the particle-wall forces from imprints on carbon paper [2] or by force sensors [3]. Each particle-wall force has to balance all interparticle forces that are exerted on the corresponding particle from above-see Fig. 1. This means that experiments essentially measure a combination of forces that we refer to as the weights of the bottom particles. For sim- plicitly, we will focus on frictionless spheres for which these weights are defined as

$$
W_{j} \equiv m_{j} g+\sum_{\langle i\rangle}\left(\vec{F}_{i j}\right)_{z}
$$

Here $m_{j}$ denotes mass, $g$ denotes gravity, $\vec{F}_{i j}$ are the interparticle forces, and $n_{c}$ is the number of particles exerting a force on particle $j$ from above; the sum runs over all these forces. There are $n_{c}$ particles excerting a force on particle $j$ from above, so the sum has $n_{c}$ terms. So, to relate the experimental results to the bulk force distributions, one has to understand the relation between weights and forces.

In this paper we will show how the local packing geometry plays the crucial role in the relation between the force distributions $P(f)$ and the weight distributions $\mathcal{P}(w)$ (we define $f=F /\langle F\rangle$ and $w=W /\langle W\rangle$ as the appropriately rescaled forces and weights). Our central point is that while the distribution of $f$ is robust, the distribution of $w$ is profoundly influenced by the contact geometry, in particular by the number of downward pointing contact forces $n_{c}$. In s.imulations of Hertzian sphere packings we will find that $\mathcal{P}_{\text {boundary }}(w)$ is different from $\mathcal{P}_{\text {bulk }}(w)$, due to the rather special packing geometry near a boundary. However, for many (but not all) experimentally relevant situations, the special packing geometry near a boundary makes $\mathcal{P}_{\text {boundary }}(w)$ rather close, but not equal, to the bulk $P(f)$. This fortunate but nontrivial coinci-
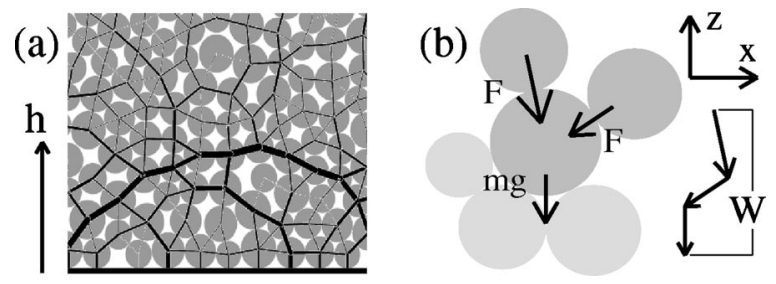

FIG. 1. (a) Detail of a typical packing in our simulations; the height $h$ denotes the distance from the bottom. The force network is represented by the black lines whose thickness is proportional to the force magnitude. (b) Definition of interparticle forces $F$ and weight $W$, for a frictionless particle with $n_{c}=2$; see Eq. (1). 
dence can be understood easily within our framework. We will, however, also provide two examples where $\mathcal{P}_{\text {boundary }}(w)$ and bulk $P(f)$ are significantly different.

Additional motivation for studying the relation between forces, weights, and geometry comes from the $q$ model [5]. Once the distinction between forces and weights has been made, one notices that the $q$ model is a lattice model in which weights are randomly redistributed over a fixed number of supporting grains. The $q$ model displays a weight distribution that is qualitatively different from both experimentally observed weight distributions and numerically obtained force distributions. We will show that this is due to the fixed connectedness of the $q$ model. Realistic $\mathcal{P}(w)$ can be obtained if we allow for the connectivity to vary within the $q$ model-e.g., by introducing random connectivity.

Our work then serves three purposes. First of all, it helps to interpret data obtained by measurements of particle-wall forces: this paper includes a section where we explicitly apply our framework to recent experimental data of highly compressed packings [11]. Second, it shows how the simple $q$ model can be extended to obtain very realistic weight distributions for both regular and irregular packings. Since the model is known to give incorrect predictions of spatial propagation [12], our intention is not to fine-tune the model and its parameters, but rather to indicate how the contact geometry is essential to describe force and weight fluctuations in more realistic packings. Third, we address the question of how force fluctuations build up as a function of the distance beneath the top surface, providing another fundamental test for theoretical models.

The paper is organized as follows. In Sec. II we first explain our numerical method and then discuss the force distributions observed in amorphous packings: it turns out that $P(f)$ is rather insensitive to the packing geometry. We then show in Sec. III that the weight distributions $\mathcal{P}(w)$, on the other hand, are very sensitive to the packing geometry. Using simple phase-space considerations, we relate $\mathcal{P}(w)$ to $P(f)$ for a given geometry. This provides a recipe how to reconstruct the bulk $P(f)$ from the experimental data, and in Sec. IV we explicitly apply this to recent experimental data on highly compressed packings [11]. In particular, our analysis strongly suggests that $P(f)$ is essentially unaffected by the tremendous deformations encountered in the experiments. We then indicate some limitations of our framework in Sec. $\mathrm{V}$, where we address subtle packing problems like the effect of gravity. In Sec. VI we investigate to what extent the $q$ model can describe the results of the numerical packings of Hertzian spheres: we derive a surprising exact result for the bond quantities $q w$, and we investigate the role of disorder in the packing geometry. Finally, we address the top-down relaxation of force fluctuations in Sec. VII. We find no evidence in the Hertzian sphere packings for the power-law relaxation predicted by the $q$ model, indicating that the model is not able to capture this spatial aspect of the force network. The paper ends with a discussion.

\section{STATISTICS OF INTERPARTICLE FORCES}

In this section we study the distribution of interparticle forces via simulations of 2D packings of frictionless spheres.
After introducing our numerical method in Sec. II A, we discuss the similarities between $P(f)$ in the bulk and near the boundary (Sec. II B). We also study the angular distribution and the probability distribution of the $z$ components of the contact forces in Sec. II C and close with a brief summary of results in Sec. II D.

\section{A. Numerical method and parameters}

Our two-dimensional packings consist of frictionless spheres (3D) under gravity. The packings are created from molecular dynamics simulations of spheres that interact through normal Hertzian forces, where $F \propto \delta^{3 / 2}$ and $\delta$ denotes the overlap distance [13]. Since Hertz's law for 2D disks is linear in $\delta$, we use $3 \mathrm{D}$ spheres. These particles reside in a container that is 24 particle diameters wide, with periodic boundary conditions in the horizontal direction. The bottom support is rigid and also has a frictionless Hertzian interaction with the particles. We construct our stationary packings by letting the particles relax from a gaslike state by introducing a dissipative force that acts whenever the overlap distance is nonzero. In this paper we use two different polydispersities: the radii $r$ are drawn from a flat distribution between either $0.49<r<0.51$ or $0.4<r<0.6$. The masses are proportional to the radii cubed. In the former case of almost monodisperse particles, the particles tend to crystallize into a triangular lattice (Sec. IV A), whereas the more polydisperse particles lead to amorphous packings such as shown in Fig. 1(a). This allows us to study how the packing geometry affects the force network. The results shown in this paper are obtained with particles that deform $0.1 \%$ under their own weight. Simulations of harder particles (deformation $0.01 \%$ ) gave similar results as those shown here [14].

The various data were obtained from 1100 realizations containing 1180 particles each. We study the force and weight distributions at various heights $h$. To do so, we divide each packing into horizontal slices of one particle diameter thickness and rescale all forces and weights in each layer to the corresponding average (absolute) values. The rescaled interparticle forces and weights will be denoted by $\vec{f}$ and $w$, respectively, with distributions $P(\vec{f})$ and $\mathcal{P}(w)$.

\section{B. Absolute values of $\vec{f}: P(f)$}

We first analyze the statistics of the absolute values $f$ $=|\vec{f}|$, whose probability density function $P(f)$ is usually referred to as the distribution of (interparticle) forces; our main finding will be that $P(f)$ in bulk and near the boundary are very similar. In Fig. 2(a) we show $P(f)$ as measured in the bulk of the amorphous packings (particle radii between $0.4<r<0.6$ ). At different heights between $10<h<30, P(f)$ was not observed to change; the open circles represent an average over these various heights. Even very close to the bottom support, we find that $P(f)$ remains almost unchanged: the dotted data set has been obtained from the forces between the bottom particles and the particles in the layer above. We refer to these forces as layer-to-layer forces near the bottom-see Fig. 2(b). So, although the bottom wall locally 

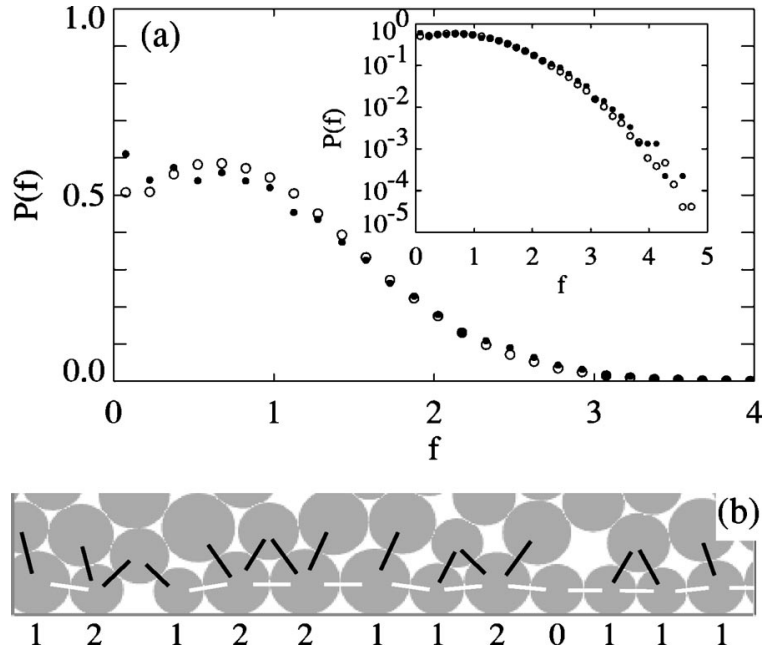

FIG. 2. (a) $P(f)$ for amorphous packing in the bulk (open circles) and for the layer-to-layer forces near the bottom (dots); the inset shows $P(f)$ on a log-lin scale. Note that the force distributions are very similar, except for a small difference for small $f$. (b) Detail of a typical packing near the bottom showing layer-to-layer forces (black lines) and the intralayer forces (white lines) near the bottom. It is clear that the layer-to-layer forces are dominant in determining the weights $w$ of the bottom particles. The numbers show the values of $n_{c}$, the number of (layer-to-layer) forces that contribute to these weights.

alters the packing geometry, the shape of $P(f)$ is essentially unaffected.

As can be seen from the inset of Fig. 2, the probability density decays slightly faster than exponentially. This is consistent with simulations by Makse et al. [15] who found that $P(f)$ crosses over to a Gaussian for large particle deformations; we have used rather "soft" particles in our simulations for which deformations are relatively large-i.e., up to $2 \%$. We come back to the effect of deformation in experiments in Sec. IV B. For small forces, $P(f)$ approaches a finite value.

\section{Orientations of $\vec{f}$ and $P^{\prime}\left(f_{z}\right)$}

After studying the absolute values of $\vec{f}_{i j}$, let us investigate the orientations of the interparticle forces. We therefore define $\varphi_{i j}$ as the angle between $\vec{f}_{i j}$ and the horizontal axis. In Fig. 3(a) we show the scatter plot of $\left(f_{i j}, \varphi_{i j}\right)$ in the bulk: the angles are uniformly distributed and independent of the absolute value of $\vec{f}$. So the packings are highly disordered away from the bottom. Near the boundary, however, this isotropy is broken strongly. The presence of the bottom wall aligns the bottom particles and as a consequence their interparticle forces become almost purely horizontal-see Fig. 2(b). It is clear that near the bottom the interparticle forces naturally divide up into these almost horizontal intralayer forces and layer-to-layer forces connecting bottom particles with those in the layer above. The orientations of these layer-to-layer forces are indeed concentrated around $\pi / 3$ and $2 \pi / 3$, as can be seen from Figs. 2(b) and 3(b).

Since the particle weights are derived from the $z$ components of the forces, $f_{z}=\left(\vec{f}_{i j}\right)_{z}$, we now investigate their distri-
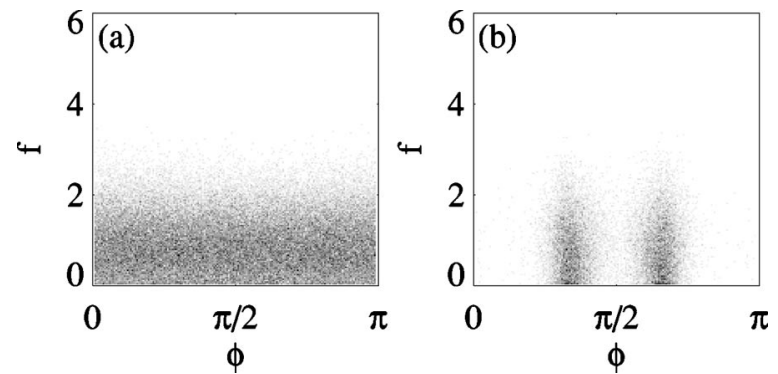

FIG. 3. Scatter plot of $\left(f_{i j}, \varphi_{i j}\right)$ for (a) the bulk forces, and (b) the layer-to-layer forces near the bottom in the amorphous packings; $\varphi_{i j}$ is the angle between the horizontal axis and the vector $\vec{f}_{i j}$.

bution $P^{\prime}\left(f_{z}\right)$. The bottom-induced orientational order discussed above is reflected in the statistics of the $f_{z}$. According to Fig. 4, there is a substantial difference between $P^{\prime}\left(f_{z}\right)$ in the bulk (open circles) and $P^{\prime}\left(f_{z}\right)$ for the layer-to layer forces near the bottom (dots). This difference can be understood as follows. Assuming that the $\varphi_{i j}$ are indeed uncorrelated to the $f_{i j}$, we can write

$$
P^{\prime}\left(f_{z}\right)=\int_{0}^{\pi} d \varphi \Phi(\varphi) \int_{0}^{\infty} d f P(f) \delta\left(f_{z}-f \sin (\varphi)\right),
$$

where $\Phi(\varphi)$ is the angle distribution and $P(f)$ is the distribution of the absolute values $|\vec{f}|$ of Fig. 2. Note that $\left\langle f_{z}\right\rangle<1$. For the layer-to-layer forces near the bottom, we have seen from the scatter plot that the values of $\sin (\varphi)$ are concentrated around $\frac{1}{2} \sqrt{3} \approx 0.866$. In the approximation that the distribution of $\sin (\varphi)$ is sharply peaked, the shape of $P^{\prime}\left(f_{z}\right)$ equals that of $P(f)$ (up to a scale factor). This is indeed confirmed by direct comparison of the dotted data sets of Figs. 2 and 4.

In the bulk, we have seen that the packing geometry is isotropic. A consequence of this isotropy is that the probability density function of the horizontal components, $P^{\prime}\left(f_{x}\right)$, is identical to $P^{\prime}\left(f_{z}\right)$ (not shown here). Again, one can use Eq.

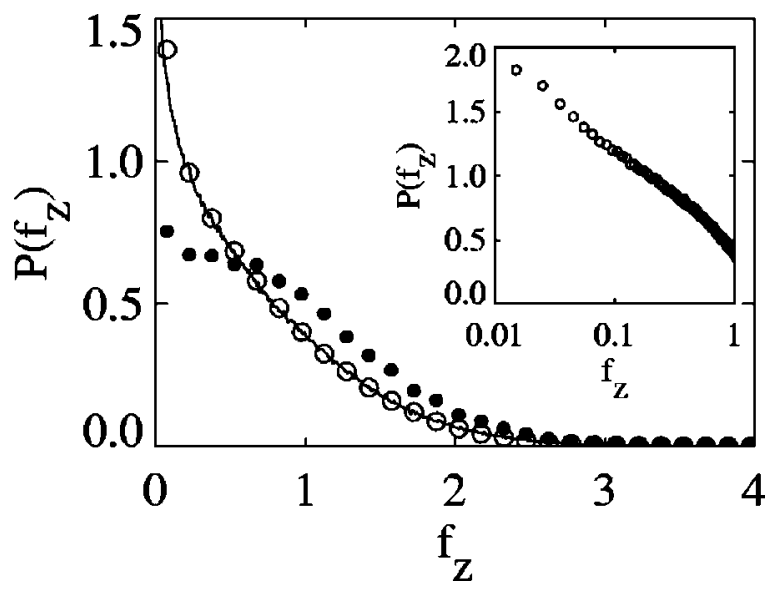

FIG. 4. $P^{\prime}\left(f_{z}\right)$ in the bulk (open circles) and for the layer-tolayer forces (dots). The solid line was obtained by numerical integration of Eq. (3). The inset shows $P^{\prime}\left(f_{z}\right)$ versus $\log f_{z}$, confirming the logarithmic divergence for small $f_{z}$. 
(2) to understand the shape of $P^{\prime}\left(f_{z}\right)$. Taking a uniform angle distribution $\Phi(\varphi)=1 / \pi$, we obtain (Appendix A)

$$
P^{\prime}\left(f_{z}\right)=\frac{2}{\pi} \int_{f_{z}}^{\infty} d f \frac{P(f)}{\sqrt{f^{2}-f_{z}^{2}}}
$$

Numerical integration of this equation with $P(f)$ from Fig. 2 yields the solid line in Fig. 4, which closely corresponds to the $P^{\prime}\left(f_{z}\right)$ as measured in the bulk (open circles). In Appendix A, we show that the integral of Eq. (3) is weakly divergent for small $f_{z}$ :

$$
P^{\prime}\left(f_{z}\right)=-\frac{2}{\pi} P(0) \ln \left(f_{z}\right)+O(1)
$$

The inset of Fig. 4 shows that our data for $P^{\prime}\left(f_{z}\right)$ is indeed consistent with this logarithmic divergence.

\section{D. $P(f)$ : Summary}

Let us briefly summarize the results of this section. The geometrical constraint imposed by the bottom wall locally induces a packing geometry which is different from the bulk geometry. Whereas this is strongly reflected in the orientations of the $\vec{f}_{i j}$, the distribution of the absolute values $P(f)$ is very robust. The probabilities for the components of the $\vec{f}_{i j}$ can be obtained with great precision, including the logarithmic divergence, by the transformation of Eq. (2).

\section{PACKING GEOMETRY AND WEIGHT DISTRIBUTIONS $\mathcal{P}(w)$}

In this section, we demonstrate that the local packing geometry has a dramatic effect on the weight distribution of $\mathcal{P}(w)$. As stated in the Introduction, experiments can only measure the particle-wall forces at the boundary of a granular packing, and not the interparticle (bulk) forces that were discussed in the previous section. Since these particle-wall forces are essentially equal to the weights of the bottom particles, it is important to understand the relation between the weight distribution $\mathcal{P}(w)$ and the distribution of interparticle forces $P(\vec{f})$. In the first part of this section we develop a simple geometrical framework to understand this relation, based on phase-space considerations. We then show that this explains, to a large extent, the weight distributions $\mathcal{P}(w)$ as measured in our simulations of Hertzian spheres. In particular, we observe substantial differences between weight distributions for different packing geometries.

\section{A. Geometrical framework: Decomposition of $\mathcal{P}(w)$ according to number of contacts $n_{c}$ from above}

If we interpret Eq. (1) as a transformation of stochastic variables, it is possible to relate the corresponding probability density functions as

$$
\begin{aligned}
\mathcal{P}_{n_{c}}(W)= & \int_{0}^{\infty} d\left(\vec{F}_{1}\right)_{z} \cdots \int_{0}^{\infty} d\left(\vec{F}_{n_{c}}\right)_{z} P\left(\left(\vec{F}_{1}\right)_{z}, \ldots,\left(\vec{F}_{n_{c}}\right)_{z}\right) \\
& \times \delta\left(W-\sum_{i=1}^{n_{c}}\left(\vec{F}_{i}\right)_{z}\right) .
\end{aligned}
$$

Here, we have neglected the term $m g$, since $m g /\langle W\rangle \ll 1$ far below the top surface of the packing. The number of forces over which we integrate differs from grain to grain, and it turns out to be crucial to label the weight distribution in Eq. (5), $\mathcal{P}_{n_{c}}(W)$, according to this number $n_{c}$. This can be seen as follows. The $\delta$ function constrains the integral on an $\left(n_{c}\right.$ -1) dimensional hyperplane of the total phase space, and the "area" of this hyperplane scales as $W^{n_{c}-1}$. We thus anticipate the following scaling behavior for small weights:

$$
\mathcal{P}_{n_{c}}(W) \propto W^{n_{c}-1} \text { for } w \rightarrow 0,
$$

provided that the joint probability density approaches a finite value when all $\left(\vec{F}_{i}\right)_{z} \rightarrow 0$. Such scaling is also implicit in the $q$ model [5], although there $n_{c} \geqslant 2$ so that $\mathcal{P}(0)=0$. The particles that do not feel a force from above, $n_{c}=0$, give a $\delta$-like contribution at $W=m g$; for deep layers this occurs for $m g /\langle W\rangle \ll 1$. In a disordered packing, the number of particles that exert a force from above can vary from grain to grain. The total weight distribution $\mathcal{P}(W)$, therefore, is a superposition of $\mathcal{P}_{n_{c}}(W)$ :

$$
\mathcal{P}(W)=\sum_{n_{c}} \rho_{n_{c}} \mathcal{P}_{n_{c}}(W),
$$

where $\rho_{n_{c}}$ is the fraction of particles with $n_{c}$ contacts from above. This means that the small weight behavior of $\mathcal{P}(W)$ depends very much on the fractions $\rho_{n_{c}}$ and thus on the local packing geometry, via Eqs. (6) and (7).

The steepness of the tail of the total weight distribution depends strongly on $\rho_{n_{c}}$ as well. To explain this, let us assume that all vertical forces $F_{z}$ contributing to the weight are uncorrelated. We consider $P^{\prime}\left(f_{z}\right) \propto e^{-\alpha f_{z}}$-i.e., $P^{\prime}\left(F_{z}\right)$ $\propto e^{-\alpha F_{z} /\left\langle F_{z}\right\rangle}$ for large forces. It follows from Eq. (5) that the weight distribution takes over this same exponent $\alpha /\left\langle F_{z}\right\rangle$, so that $\mathcal{P}_{n_{c}}(W) \propto e^{-\alpha W /\left\langle F_{z}\right\rangle}$. However, the $\mathcal{P}_{n_{c}}(W)$ 's are not properly normalized: $\langle W\rangle_{n_{c}}=\left\langle F_{z}\right\rangle n_{c}$, since each of the $F_{z}$ gives an average contribution $\left\langle F_{z}\right\rangle$. This yields a total average weight $\langle W\rangle=\left\langle F_{z}\right\rangle \Sigma_{n_{c}} \rho_{n_{c}} n_{c}=\left\langle F_{z}\right\rangle\left\langle n_{c}\right\rangle$. In order to compare with experimental and theoretical results we have to rescale the weights so that $\langle w\rangle=1$, yielding the following large weight behavior:

$$
\mathcal{P}(w) \propto e^{-\gamma w} \text { with } \gamma=\alpha\left\langle n_{c}\right\rangle .
$$

This simple calculation shows that, for a given value of $\alpha$, the steepness of the tail of the experimentally measured weight distribution is very sensitive to the local packing geometry. This is a direct consequence of keeping $\langle w\rangle$ fixed to unity: a decrease of probability for small weights must lead to a steeper tail for large weights in order to leave the average weight unaltered. Note that this general argument is not restricted to uncorrelated $F_{z}$ or exponential tails. A generali- 

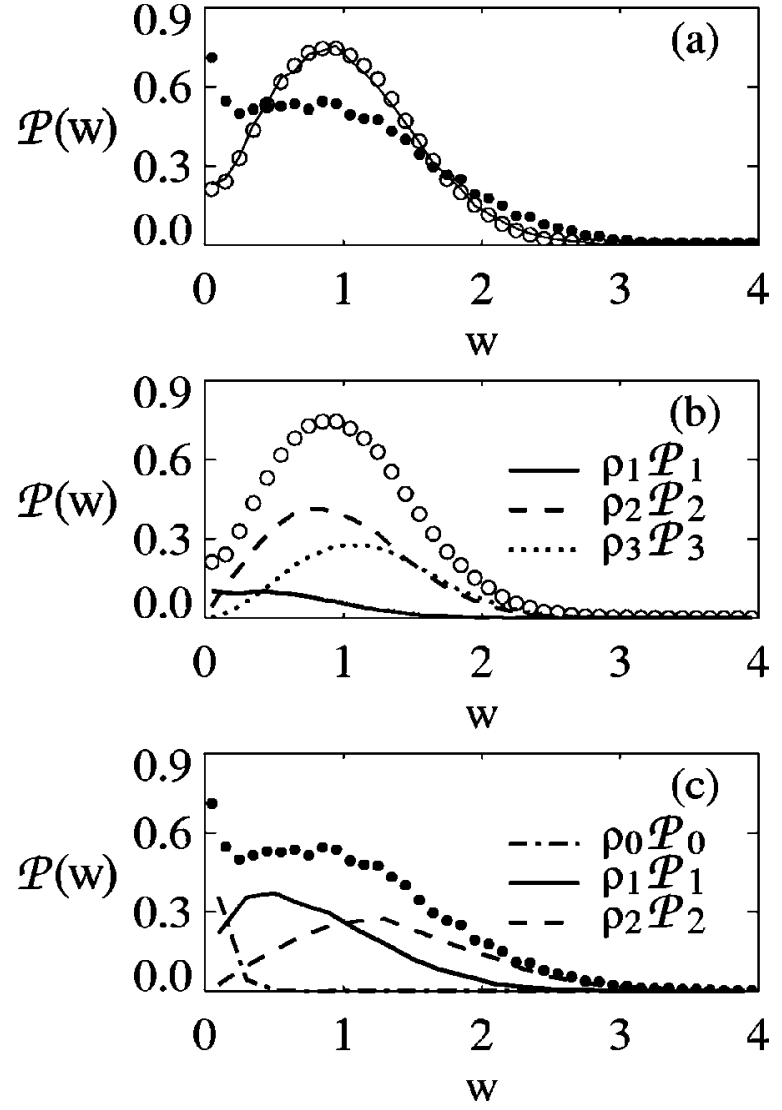

FIG. 5. (a) $\mathcal{P}(w)$ in the bulk (open circles) and at the bottom (dots) in amorphous packings. At $2<h<3, \mathcal{P}(w)$ is already bulk like (solid line). (b), (c) Decomposition of $\mathcal{P}(w)$ according to Eq. (7) (b) in the bulk (open circles) and (c) at the bottom (dots). The measured bulk values for the fractions $\left\{\rho_{0}, \rho_{1}, \rho_{2}, \rho_{3}\right\}$ in Eq. (7) are $\{0.01,0.11,0.52,0.36\}$, and the bottom values are $\{0.08,0.46,0.44,0.02\}$; as explained in [16], we excluded the intralayer (almost horizontal) forces at the bottom when determining $n_{c}$.

zation to other than exponential tails is given in Appendix B.

So we have advanced a simple picture, in which the shape of $\mathcal{P}(w)$ depends strongly on the local packing geometry via the fractions $\rho_{n_{c}}$. The small force behavior follows from Eqs. (6) and (7), whereas Eq. (8) relates to a good approximation the exponential tails of $P^{\prime}\left(f_{z}\right)$ and $\mathcal{P}(w)$. The object one ultimately wishes to characterize is of course the force distribution $P(f)$. Since close to the boundary $P(f)$ and $P^{\prime}\left(f_{z}\right)$ are identical up to a scaling factor $\left\langle f_{z}\right\rangle$ (Sec. II C), the above equations allow us to trace the features of the force distribution from experimental measurements. Along this line, we analyze recent experimental data in Sec. IV B.

\section{B. $\mathcal{P}(w)$ in Hertzian sphere packings}

We now discuss the weight distributions observed in the Hertzian sphere packings and interpret the results within the framework developed above. Figure 5(a) shows that in the amorphous packing $\mathcal{P}(w)$ in the bulk (open circles) is significantly different from $\mathcal{P}(w)$ of the bottom particles (dots). The probability for small weights is much larger at the bottom, and the decay for large weights is not as steep as for the bulk
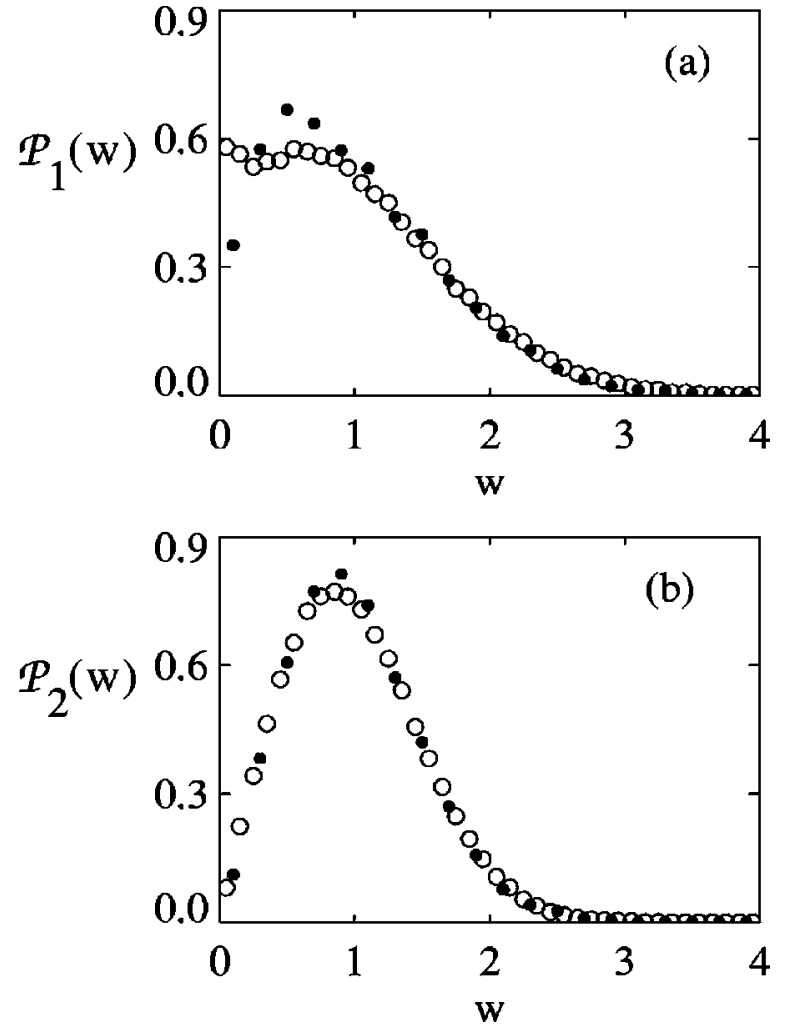

FIG. 6. Direct comparison of (a) $\mathcal{P}_{1}(w)$ and (b) $\mathcal{P}_{2}(w)$ for bulk (open circles) and bottom particles (dots). All distributions are scaled such that $\langle w\rangle=1$.

particles. Furthermore, the transition from bottom to bulk behavior is remarkably sharp: in the slice $2<h<3$ (solid curve), the weight distribution is already bulk like.

Using the concepts developed in the preceding paragraphs, we now show how this change in $\mathcal{P}(w)$ can be explained by a change in the local packing geometry. Consider the typical bottom configuration of Fig. 2(b). The intralayer forces (white lines) are almost purely horizontal and hence do not contribute to the weights. This reduces the effective values of $n_{c}$, leading to the following fractions for the bottom particles: $\left\{\rho_{0}, \rho_{1}, \rho_{2}, \rho_{3}\right\}=\{0.08,0.46,0.44,0.02\}$, where we did not count the intralayer forces for determining the values of $n_{c}$ [16]. In the bulk, these fractions are different-namely, $\left\{\rho_{0}, \rho_{1}, \rho_{2}, \rho_{3}\right\}=\{0.01,0.11,0.52,0.36\}$. According to Eq. (7), these differences between the $\rho_{n_{c}}$ in the bulk and at the bottom should lead to a substantially different $\mathcal{P}(w)$. Figures 5(b) and 5(c) explicitly show the decomposition into the $\mathcal{P}_{n_{c}}(w)$. Indeed, one observes the scaling behavior for small $w$ proposed in Eq. (6). Moreover, the various $\mathcal{P}_{n_{c}}(w)$ are essentially the same at the bottom and in the bulk: a direct comparison is given in Fig. 6, where we rescaled the average values to unity. There is only a small difference in the $\mathcal{P}_{1}(w)$ due to the fact that bottom particles with $n_{c}=1$ are typically smaller than average [Fig. 6(a)]. For these particles, the intralayer forces will add a small contribution to the weights, enhancing $\mathcal{P}_{1}(w)$ for small $w$ at the expense of $\mathcal{P}_{1}(0)$. The same argument holds for $\mathcal{P}_{0}(w)$, whose $\delta$-like shape appears a bit broadened in Fig. 5(c). However, it is clear that the 
differences between $\mathcal{P}(w)$ in the bulk and at the bottom are mainly due to a change in contact geometry.

Finally, let us remark that the good agreement between $P_{\text {bulk }}(f)$ and $\mathcal{P}_{\text {boundary }}(w)$ for $w>0.3$ is fortuitous and due to the relatively large fraction of bottom particles with $n_{c}=1$. We will argue below that this is also the case in many (but not all) carbon paper experiments.

\section{Summarizing the simple picture}

Our simple framework as developed in the sections above can be summarized as follows: The geometry of the contact network has a strong effect on $\mathcal{P}(w)$, while $P(f)$ is very robust. The weight distribution for particles with a given $n_{c}$, $\mathcal{P}_{n_{c}}(w)$, is robust and behaves as $w^{n_{c}-1}$ for small $w . \mathcal{P}(w)$ can be decomposed as $\mathcal{P}(w)=\sum_{n_{c}} \rho_{n_{c}} \mathcal{P}_{n_{c}}(w)$, where $\rho_{n_{c}}$ are the fractions of particles that have $n_{c}=0,1,2, \ldots$ "up" contacts. Differences of $\rho_{n_{c}}$ between boundary particles and bulk particles explain the different $\mathcal{P}(w)$ 's for these cases. When $\rho_{0}$ and $\rho_{1}$ are large, the total weight distribution $\mathcal{P}(w)$ exhibits a plateau at small weights and a slow decay at large weights; when $\rho_{2}$ and $\rho_{3}$ become large, $\mathcal{P}(w)$ becomes sharply peaked. In this way, the $\mathcal{P}(w)$ small weight behavior and its exponential decay rate for large weights reflect the packing geometry.

\section{MANIPULATING THE GEOMETRY: EXPERIMENTAL RELEVANCE}

So far we have focused on the role of the bottom boundary for disordered packings of frictionless particles. In this section we provide explicit examples of other types of packing geometries and their effect on $\mathcal{P}(w)$. We first discuss our simulations of weakly polydisperse particles, which give rise to rather crystalline packings-see Fig. 7(a). We then apply the geometrical framework derived in the previous section to experimental (carbon paper) data by Erikson et al. [11] of highly deformed packings of soft rubber particles. Their results have a natural interpretation within our framework and form a nice illustration of how the number of contact affects the weight distribution. Both the simulations of crystalline packings and the experiments on deformed packings are examples where the experimentally accessible $\mathcal{P}_{\text {boundary }}(w)$ is significantly different from $P(f)$ in the bulk; we discuss why in many other carbon paper experiments $\mathcal{P}_{\text {boundary }}(w)$ is probably very similar to the real $P(f)$.

\section{A. Crystalline versus disordered frictionless packings}

We now present the results of the more or less crystalline packings, obtained from simulations with particle radii between $0.49<r<0.51$. First, the force distribution $P(f)$ shown in Fig. 7(b) is indistinguishable from the force distributions in the amorphous packings [compare with Fig. 2(a)]. So, despite the order in particle positions, there are still large fluctuations in the force network. There is of course some disorder in the "contact network" since not all particles are in contact with their six neighbors [Fig. 7(a)]. It is nevertheless surprising that for this very different contact geometry, the force fluctuations are characterized by the same probability
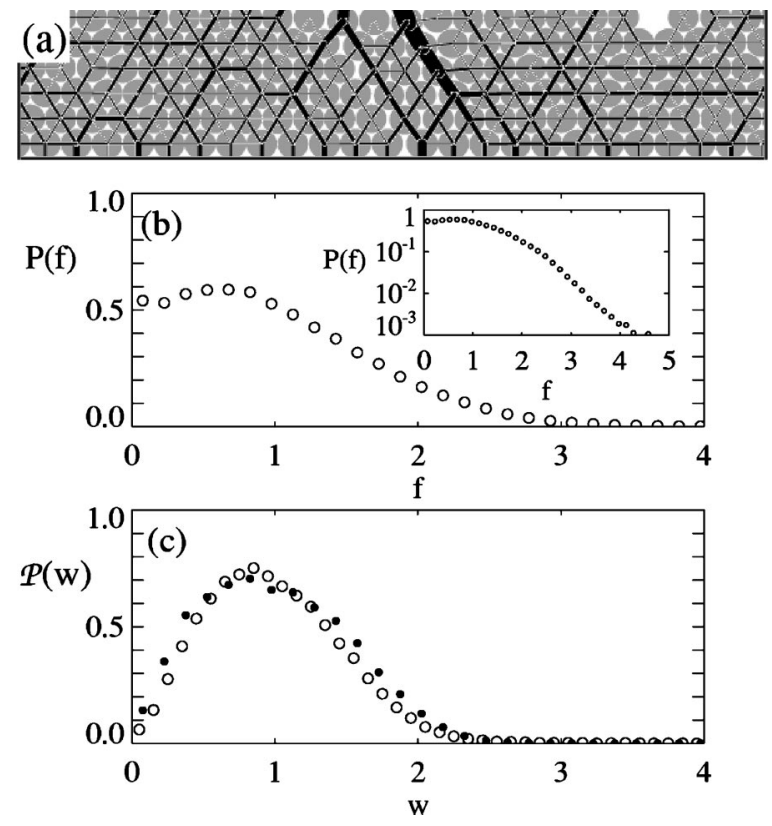

FIG. 7. (a) Weakly polydisperse particles (radii between $0.49<r<0.51$ ) spontaneously crystallize into a hexagonal packing. (b) The corresponding $P(f)$ is indistinguishable from the force distributions in amorphous packings. (c) The weight distributions $\mathcal{P}(w)$ in the bulk (open circles) and at the bottom (dots) are dominated by particles with $n_{c}=2$.

distribution as was observed for highly disordered packings. This strongly suggests that $P(f)$ is a very robust quantity and independent of the packing geometry.

The weight distribution $\mathcal{P}(w)$, on the other hand, is very sensitive to the geometry. In a perfect triangular packing all particles would have $n_{c}=2$; in our simulations we find that $\rho_{2}=0.9$ and $\rho_{1}=0.1$ due to lattice imperfections. From our geometrical framework we expect that the shape of the weight distribution is dominated by $\mathcal{P}_{2}(w)$. Figure $7(\mathrm{c})$ shows that this is indeed the case-e.g., compare with Fig. 6(b).

In an earlier paper [8], we reported how one can break the regular packing geometry by using curved boundaries. This led to a dramatic change in $\mathcal{P}(w)$ that again could be understood from a change in the $\rho_{n_{c}}$.

\section{B. Experiments on strongly deformed particles}

We now demonstrate how the strategy to decompose the weight distributions according to $n_{c}$ can be applied to experiments measuring $\mathcal{P}(w)$ at the boundary of a granular material. This is best illustrated by recent carbon paper experiments by the Chicago group on soft rubber beads, in particular Fig. 3 of Ref. [11], in which the effect of particle deformations was investigated. Although our numerical study has been done in two dimensions with frictionless particles, the general phase-space considerations presented in Sec. III A are independent of dimensionality and are therefore expected to be applicable to the experimental situation. The raw data of these experiments were kindly made avail- 
TABLE I. The calculated values for the exponents $\hat{\alpha}$, after estimating the fractions $\rho_{n_{c}}$ from the experimental data of Figs. 3(a)3(d) of Ref. [11]. The percentage in the first column represents the degree of particle deformation. The values of $\gamma$ are taken from Table I of Ref. [11].

\begin{tabular}{|c|c|c|c|c|c|c|c|}
\hline \multirow[b]{2}{*}{ Deform. } & \multirow[b]{2}{*}{$\gamma$} & \multirow[b]{2}{*}{$\rho_{0}$} & \multirow[b]{2}{*}{$\rho_{1}$} & \multicolumn{2}{|c|}{$\rho_{2}=\rho_{3}$} & \multicolumn{2}{|c|}{$\rho_{3}=0$} \\
\hline & & & & $\left\langle n_{c}\right\rangle$ & $\hat{\alpha}=\frac{\gamma}{\left\langle n_{c}\right\rangle}$ & $\left\langle n_{c}\right\rangle$ & $\hat{\alpha}=\frac{\gamma}{\left\langle n_{c}\right\rangle}$ \\
\hline $25 \%$ & 2.4 & 0.23 & 0.58 & 1.05 & 2.29 & 0.96 & 2.51 \\
\hline $30 \%$ & 2.6 & 0.21 & 0.26 & 1.60 & 1.63 & 1.33 & 1.96 \\
\hline $37 \%$ & 2.8 & 0.14 & 0.18 & 1.88 & 1.49 & 1.54 & 1.81 \\
\hline $45 \%$ & 3.8 & 0.00 & 0.05 & 2.42 & 1.57 & 1.95 & 1.95 \\
\hline
\end{tabular}

able by the authors, allowing us to perform the analysis presented below.

The experimental results of Fig. 3 of Ref. [11] display three trends as the compression is increased: (i) The $\delta$-like peak at $w=0$ decreases, (ii) $\lim _{w \downarrow 0} \mathcal{P}(w)$ decreases, and (iii) The exponential tail becomes steeper.

These behaviors emerge naturally when considering the role of the fractions $\rho_{n_{c}}$. The first trend arises from a decrease in $\rho_{0}$, since only particles with $n_{c}=0$ give a $\delta$-like contribution to $\mathcal{P}(w)$. The second trend comes from a decrease in $\rho_{1}$ : from Eqs. (6) and (7) it is clear that $\lim _{w \downarrow 0} \mathcal{P}(w)=\rho_{1} \mathcal{P}_{1}(w)$. The changes in $\mathcal{P}(w)$ can thus be understood from an increasing number of contacts, which is what one would expect for a compressed system [15]. The fractions $\rho_{2}$ and $\rho_{3}$ will increase at the expense of $\rho_{0}$ and $\rho_{1}$. Also the third trend, the steepening of the exponential tail, is directly related to the increase in $\left\langle n_{c}\right\rangle$ via Eq. (8). However, Eqs. (6)-(8) allow us to further quantify this change in contact geometry from the experimental data. The value of $\rho_{1} \mathcal{P}_{1}(0)$ can be read off from the plots, after subtracting the $\delta$-like data points, since $\rho_{1} \mathcal{P}_{1}(0)=\lim _{w \downarrow 0} \mathcal{P}(w)$. The value of $\rho_{0}$ is obtained by the height of the $\delta$ peak times the bin width. Using the raw experimental data, we obtained the figures given in the first colomn of Table I, where we took $\mathcal{P}_{1}(0)=0.5$ [17]. Unfortunately, the values of $\rho_{2}$ and $\rho_{3}$ cannot be determined directly from the data.

An intriguing issue is that numerical simulations by Makse et al. [15] indicate that $P(f)$ crosses over to a Gaussian for large particle deformations. This contradicts the experimental data for which one observes an exponential tail even though particle deformations are up to no less than $45 \%$ [11]. Moreover, we speculate below that the steepening of the tails is only due to changes in the $\rho_{n_{c}}$ and that the bulk force distributions $P(f)$ actually remain unaffected by the particle deformations. The way to test this scenario is to examine whether the exponential decay constant of $P(f)$ $\propto e^{-\hat{\alpha} f}$ remains fixed, even though the steepness of $\mathcal{P}(w)$ $\propto e^{-\gamma w}$ increases. We use Eq. (8) to determine the value of $\alpha=\gamma /\left\langle n_{c}\right\rangle$, where $\alpha$ and $\gamma$ are the decay rates of $P^{\prime}\left(f_{z}\right)$ and the experimental $\mathcal{P}(w)$, respectively. Since we found in Sec. II $\mathrm{C}$ that $P(F)$ and $P^{\prime}\left(F_{z}\right)$ near the bottom are almost identical up to a scaling factor $\left\langle F_{z}\right\rangle /\langle F\rangle$, the actual decay rate of $P(f) \propto e^{-\hat{\alpha} f}$ is exactly the same as that of the (renormalized)
$P^{\prime}\left(f_{z}\right)$, so that $\hat{\alpha}=\alpha$. Hence, we can approximate the exponential decay constant of the force distribution as

$$
\hat{\alpha}=\frac{\gamma}{\left\langle n_{c}\right\rangle} .
$$

To estimate the values of $\left\langle n_{c}\right\rangle$, we worked out two scenarios: we take either $\rho_{2}=\rho_{3}$ or $\rho_{3}=0$. Together with the values of $\rho_{0}, \rho_{1}$, and $\gamma$, taken from the experimental data, this yields the values of $\hat{\alpha}$ listed in the second and third columns of Table I. Surprisingly, the root-mean-square deviation in $\hat{\alpha}$ is only $18 \%$, which is rather small considering our rather crude estimates of the $\rho_{n_{c}}$ and the fact that Eqs. (8) and (9) are only approximate.

Let us briefly recapitulate the discussion above. First, we have interpreted the changes in experimental particle-wall force distributions of strongly compressed packings [11] as a change in the packing geometry. To be more precise, the overall trends can be understood from the expected increase of the number of contacts due to compression. We demonstrated how one can determine the fractions $\rho_{0}$ and $\rho_{1}$ from the experimental data. At first sight the obtained percentages of particles with $n_{c}=0$ or 1 in Table I may appear to be rather high for 3D packings. However, one should keep in mind that in the experiment the number of bottom particles is often known, but that some particles clearly do not leave an imprint. This indicates that there is a significant number of bottom particles that really have $n_{c}=0$, even for these $3 \mathrm{D}$ systems. Clearly this issue is not settled, so direct measurements of these fractions would therefore be very welcome as a test of our framework. Furthermore, our crude estimates in Table I give reason to believe that the force distribution $P(f)$ is actually not much affected by the compression. Again, this scenario should be verified by measuring the $\rho_{n_{c}}$ more directly. Finally, it seems that for most experimental results, where particle deformations are relatively small, $\rho_{0}$ and $\rho_{1}$ are substantial at the boundary, so that $\mathcal{P}_{\text {boundary }}(w)$ is similar to $\mathcal{P}_{\text {bulk }}(f)$ (apart from a $\delta$ peak at $w=0$ ). The same argument probably holds for recent simulations by Silbert et al. [18].

\section{BEYOND THE SIMPLE PICTURE}

In the picture that we have constructed above we characterize the packing geometry by the fractions $\rho_{n_{c}}$, and we found that the $P_{n_{c}}(w)$ are very robust. This is of course a vast simplification, since we characterize the local environment of a particle by only one number-namely, $n_{c}$. In this section we address the question why this crude approach works so remarkably well. For bottom particles the situation is particularly simple and insightful, since the geometry of the contacts is more or less fixed. There is one contact with the bottom, one or two almost horizontal intralayer contacts, and $n_{c}$ forces from above-Fig. 2(b). As we have shown in Fig. 3(b), the angles of these forces display little scatter, so the local texture is more or less fixed once $n_{c}$ is given. For bottom particles one can thus understand that $n_{c}$ indeed provides a good description of the local packing geometry, which justifies the decompostion according to $n_{c}$. Although for particles in the bulk the situation is more complicated, there are 
similar arguments why $\mathcal{P}_{n}(w)$ is indeed a robust quantity -i.e., insensitive to packing geometry. These will be discussed in Sec. V A. We then address the up-down symmetry of the system. Our framework only involves the number of contacts from above, $n_{c}$, and not the number of contacts from below, $n_{b}$. For bottom particles $n_{c}$ is the obvious parameter, but in the bulk of an amorphous packing, where the angle distribution is isotropic, there is no reason why $n_{c}$ should be more important than $n_{b}$. In Sec. V B we therefore investigate weight distributions for particles with a given combination $\left\{n_{c}, n_{b}\right\}$, which we denote by $\mathcal{P}_{n_{c} n_{b}}(w)$. Special attention will be paid to particles that have $n_{c} \neq n_{b}$ in Sec. V C.

\section{A. Why is $\mathcal{P}_{\boldsymbol{n}_{\boldsymbol{c}}}(\boldsymbol{w})$ for bulk particles robust?}

It is not a priori clear why $\mathcal{P}_{n_{c}}(w)$ is rather insensitive for the packing geometry, since the definition of $\mathcal{P}_{n_{c}}(w)$ in Eq. (5) involves the joint distribution of the $\left(\vec{f}_{i}\right)_{z}$ that push on a particle from above-i.e., $P\left(\left(\vec{f}_{1}\right)_{z}, \ldots,\left(\vec{f}_{n_{c}}\right)_{z}\right)$. This joint distribution has an explicit geometry dependence since the projections in the $z$ direction involve the distribution of contact angles $\varphi_{i}$. Even if we assume that the force magnitude is uncorrelated to its orientation, i.e.,

$$
P\left(\vec{f}_{1}, \ldots, \vec{f}_{n_{c}}\right)=P\left(f_{1}, \ldots, f_{n_{c}}\right) \Phi\left(\varphi_{1}, \ldots, \varphi_{n_{c}}\right),
$$

we obtain the distribution of the vertical components $P\left(\left(\vec{f}_{1}\right)_{z}, \ldots,\left(\vec{f}_{n_{c}}\right)_{z}\right)$ by integration over the joint angle distribution $\Phi\left(\varphi_{1}, \ldots, \varphi_{n_{c}}\right)$. Therefore, the $\mathcal{P}_{n_{c}}(w)$ have an explicit geometry dependence.

We already saw that this angle distribution is more or less fixed for bottom particles. For the polydispersities used in this study, the bulk angles have also limited room for fluctuations once $n_{c}$ has been specified. For example if $n_{c}=3$, one typically finds one angle close to $\pi / 2$ and two relatively small angles-see Fig. 8(a); this is because the three particles should all touch the upper half of the bead supporting them. Particles with $n_{c}=2$ also have such an "excluded-volume"like constraint [Fig. 8(b)], albeit less strong than for $n_{c}=3$. Particles with $n_{c}=1$ have an enhanced probability for angles around $\pi / 2$, because such contacts make the presence of a second contact from above less probable [Fig. 8(c)]. So the shape of $\mathcal{P}_{n_{c}}(w)$ is limited by the geometric constraints on the angle distributions $\Phi\left(\varphi_{1}, \ldots, \varphi_{n_{c}}\right)$, which are rather peaked. This justifies the picture that the geometry dependence of $\mathcal{P}(w)$ is mainly due to the $\rho_{n_{c}}$ and that the $\mathcal{P}_{n_{c}}(w)$ can be considered invariant.

Note that the above-mentioned constraints on the angle distributions imply that the averages $\langle w\rangle_{n_{c}}$ are not simply proportional to $n_{c}$. Comparing, for example, $n_{c}=1$ and $n_{c}$ $=3$, we see that the two "extra" forces for $n_{c}=3$ have a relatively small vertical component; the average weight will thus grow less than linearly with $n_{c}$. We should therefore correct Eq. (8) for the steepness of the tails by replacing $\left\langle n_{c}\right\rangle$ with $\Sigma_{n_{c}} \rho_{n_{c}}\langle w\rangle_{n_{c}}$. Making a correction of this type would further refine our analysis of the experiment with rubber beads discussed in Sec. IV B.
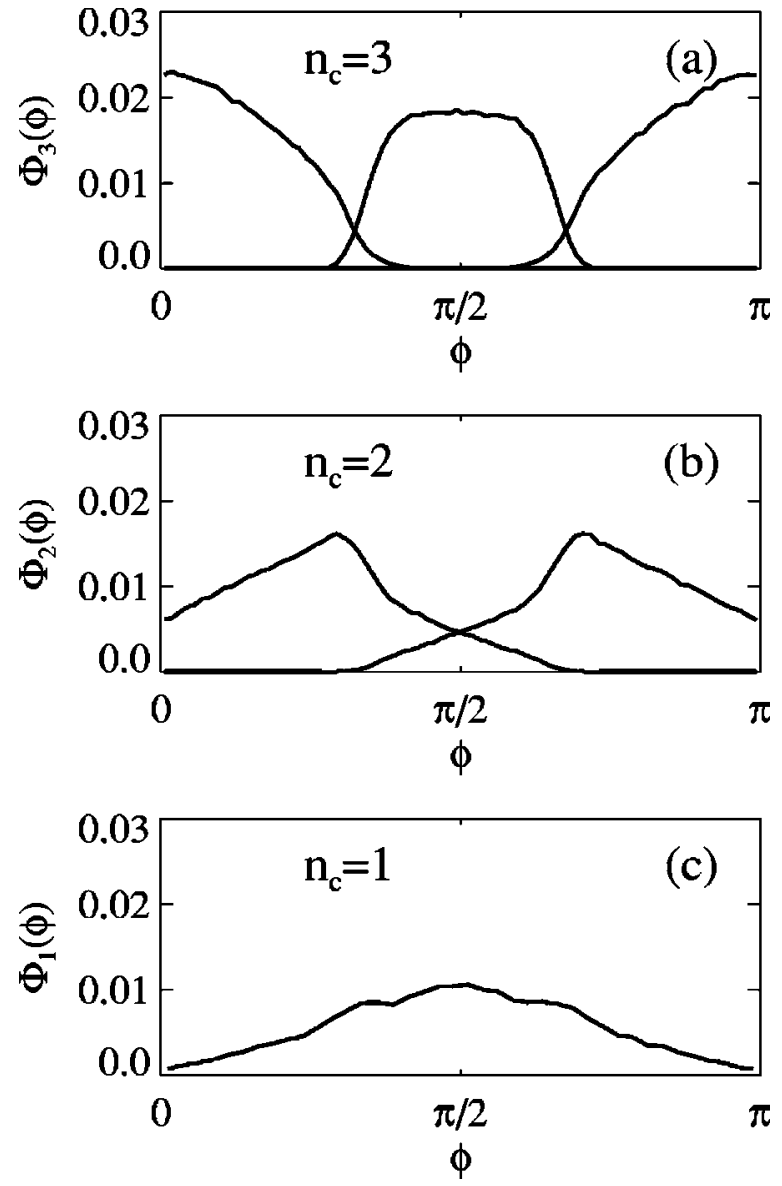

FIG. 8. (a) For particles with $n_{c}=3$, we plot the probability densities for the angles $\Phi_{3}\left(\varphi_{1}\right), \Phi_{3}\left(\varphi_{2}\right)$, and $\Phi_{3}\left(\varphi_{3}\right)$, where the three angles have been sorted such that $\varphi_{1}<\varphi_{2}<\varphi_{3}$. (b) The probability densities $\Phi_{2}\left(\varphi_{1}\right)$ and $\Phi_{2}\left(\varphi_{2}\right)$ for particles with $n_{c}=2$. (c) The probability density $\Phi_{1}\left(\varphi_{1}\right)$ for particles with $n_{c}=1$.

\section{B. Gravity and up-down symmetry}

In our analysis of $\mathcal{P}(w)$ we have explicitly broken the up-down symmetry, since it only involved the number of contacts from above. At the bottom, this is an obvious choice. Away from the boundary, however, the amorphous packings have an isotropic angle distribution even though the packings were created under gravity. Moreover, we have neglected the term $m g$ in Eq. (1), which makes the sum of forces from below equal to the sum of forces from above. So in principle one could also decompose $\mathcal{P}(w)$ according to the number of contacts from below $n_{b}$. We therefore investigate $\mathcal{P}_{n_{c} n_{b}}(w)$; this can be regarded as a "component" of $\mathcal{P}_{n_{c}}(w)$, since $\rho_{n_{c}} \mathcal{P}_{n_{c}}(w)=\sum_{n_{b}} \rho_{n_{c} n_{b}} \mathcal{P}_{n_{c} n_{b}}(w)$.

Figure 9(b) shows that $\mathcal{P}_{13}(w), \mathcal{P}_{22}(w)$, and $\mathcal{P}_{31}(w)$ are almost identical. The same holds for $\mathcal{P}_{23}(w)$ and $\mathcal{P}_{32}(w)$ [Fig. 9(c)], so the total coordination number $n_{c}+n_{b}$ appears to be a more fundamental quantity than just $n_{c}$ or $n_{b}$. Figure 9(d) furthermore shows that the quadratic scaling of $\mathcal{P}_{33}(w)$ is somewhat more pronounced than for $\mathcal{P}_{23}(w)$ and $\mathcal{P}_{32}(w)$; it seems that the presence of two contacts from above or below inhibits the pure quadratic scaling.

The presence of gravity is noticed, however, for $\mathcal{P}_{12}(w)$ and $\mathcal{P}_{21}(w)$ which do show some differences [Fig. 9(a)]. 

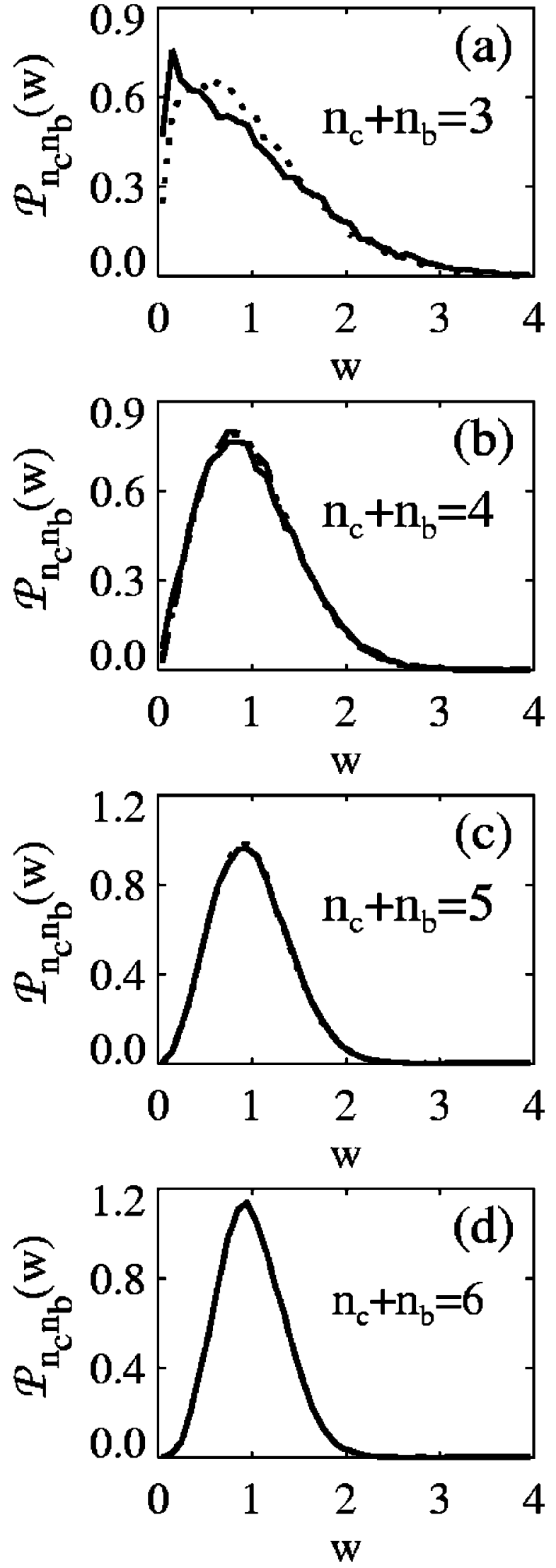

FIG. 9. (a) $\mathcal{P}_{12}(w)$ (solid line) and $\mathcal{P}_{21}(w)$ (dotted line). (b) $\mathcal{P}_{13}(w), \mathcal{P}_{22}(w)$, and $\mathcal{P}_{31}(w)$. (c) $\mathcal{P}_{23}(w)$ and $\mathcal{P}_{32}(w)$. (d) $\mathcal{P}_{33}(w)$.
TABLE II. Fractions $\rho_{n_{c} n_{b}}$ expressed in percentages; the numbers are almost up-down symmetric, except for rattlers (particles with two contacts). From these fractions one finds the average coordination number $\left\langle n_{c}+n_{b}\right\rangle=4.51$.

\begin{tabular}{cllccl}
\hline \hline$n_{c} \backslash n_{b}$ & 0 & 1 & 2 & 3 & \multicolumn{1}{c}{4} \\
\hline 0 & 0 & 0 & 0.6 & 0 & 0 \\
1 & 0 & 0.3 & 5.6 & 4.7 & 0.2 \\
2 & 0 & 4.7 & 26.1 & 20.5 & 0.7 \\
3 & 0 & 5.1 & 21.6 & 8.9 & 0 \\
4 & 0 & 0.3 & 0.7 & 0 & 0 \\
\hline \hline
\end{tabular}

These particles have only three contacts and were less restricted during the formation of the static force network by the "cage" surrounding them. This allowed gravity to influence their final movements more than for particles with $n_{c}$ $+n_{b}>3$. Obviously, this effect is even stronger for particles with only two contacts, which typically have $\left\{n_{c}, n_{b}\right\}=\{0,2\}$.

To further investigate the up-down symmetry, we list the fractions $\rho_{n_{c} n_{b}}$ of particles with a certain $n_{c}$ and $n_{b}$ in Table II. For all particles with three or more contacts these fractions are almost perfectly symmetric. From this we conclude that in the amorphous packings, the up-down asymmetry due to gravity is only noticed by particles that have two or three contacts.

\section{Particles with $n_{c} \neq n_{b}$}

We have seen that for particles with $\left\{n_{c}, n_{b}\right\}=\{3,1\}$ or vice versa, the small weight behavior is $\sim w^{1}$, which is different from the scaling predicted by Eq. (6). This breakdown of our simple picture can be understood as follows. A particle that has four contacts can either have $\left\{n_{c}, n_{b}\right\}=\{3,1\},\left\{n_{c}, n_{b}\right\}$ $=\{2,2\}$, or $\left\{n_{c}, n_{b}\right\}=\{1,3\}$ depending on the precise orientations of the forces with respect to gravity. However, if we were to define the weights by projecting the $\vec{F}_{i j}$ at a small angle with respect to gravity, a particle with four contacts can easily change from $\left\{n_{c}, n_{b}\right\}=\{3,1\}$ to $\{2,2\}$ or even to $\{1,3\}$. However, we have seen that there is no "preferred" projection direction, since gravity has only very little effect on our packings. Hence, it is not surprising that the $\mathcal{P}_{n_{b} n_{c}}(w)$ depend on $n_{c}+n_{b}$ and not on $n_{c}$ or $n_{b}$ individually.

But what determines the precise scaling for small weights? Consider a particle $i$ with $n_{c}=3$ and $n_{b}=1$. The three forces pushing it from above, $\vec{F}_{i 1}, \vec{F}_{i 2}$ and $\vec{F}_{i 3}$, are not independent: force equilibrium in the direction perpendicular to $\vec{F}_{i 4}$ (the force pushing from below) requires $\left(\vec{F}_{i 1}+\vec{F}_{i 2}\right.$ $\left.+\vec{F}_{i 3}\right) \cdot \vec{e}_{\perp}=0$, where $\vec{F}_{i 4} \cdot \vec{e}_{\perp}=0$. This reduces the number of independent forces from above to only 2 , since the third is determined by mechanical equilibrium. As a consequence, the scaling behavior for small $w$ will be $\mathcal{P}_{31}(w) \propto w$.

For particles with $n_{c}=3$ and $n_{b}=2$, the five forces are also coupled through mechanical equilibrium. In this case, however, one cannot distill a relation between the forces from above only, such as we did for particles with $\left\{n_{c}, n_{b}\right\}$ $=\{3,1\}$. So one still expects that $\mathcal{P}_{32}(w) \propto w^{2}$, as is observed 
in Fig. 9(c). Nevertheless, this illustrates that twodimensional mechanical equilibrium does introduce correlations between all forces pushing from above. This limits the validity of our arguments used in Sec. III, for bulk particles. At the bottom our analysis is still valid: horizontal equilibrium can be accomplished by the forces between neighboring bottom particles [see Fig. 2(b)], so the forces from above can really be considered as independent.

\section{Summary}

In this section we have addressed the limitations of our simple geometrical framework. We have shown that the observation that $\mathcal{P}_{n_{c}}(w)$ is insensitive to packing geometry originates from excluded-volume-like correlations between the angles at which forces press upon a bead (Fig. 8). This is the subtle underlying reason why our simple picture, where we characterize the local packing geometry by only one number $n_{c}$, is good enough to interpret experimental and numerical data. We have furthermore studied the effect of gravity by decomposing the weight distribution according to the number of particles from below $\left(n_{b}\right)$ as well. We found that gravity breaks the up-down symmetry only mildly in our simulations; the distributions $\mathcal{P}_{n_{c} n_{b}}(w)$ depend on the coordination number $n_{c}+n_{b}$ rather than on $n_{c}$ or $n_{b}$ independently (Fig. 9). This further refines the analysis of the relation between packing geometry and force network statistics in the bulk of a packing; at the boundary, it is sufficient to consider only the number of contacts from above $\left(n_{c}\right)$.

\section{WEIGHT AND FORCE DISTRIBUTIONS IN THE $q$ MODEL: THE ROLE OF CONNECTIVITY}

In this section, we investigate to what extent the results obtained for the Hertzian sphere packings can be understood within the context of the $q$ model and its generalizations. In the standard version of the model, the particles are positioned on a regular lattice, and the particle weights are stochastically transmitted to the neighbors in the layer below [5]. The weight on a particle $i$ splits up into $n_{c}$ fractions $q_{i j}$, and the total weight exerted on a particle $j$ in the layer below then becomes

$$
W_{j}=m g+\sum_{i} q_{i j} W_{i}
$$

where the term $m g$ can be neglected at large depth. The fractions $q_{i j}$ obey the constraint

$$
\sum_{j} q_{i j}=1
$$

which assures mechanical equilibrium in the vertical direction. In both Eqs. (11) and (12), the sum runs over $n_{c}$ terms. These $q_{i j}$ can in principle be deduced from the forces in more realistic packings: from definition (1), one finds $q_{i j}$ $=\left(\vec{F}_{i j}\right)_{z} / W_{i}$.

The simple form of the $q$ model has allowed for a number of exact results of which the most important is the solution for the uniform $q$ distribution. This uniform $q$ distribution assigns an equal probability to each set of $\left\{q_{i j}\right\}$ that obeys Eq. (12) and serves as a generic case. The rescaled weights $w$ then become distributed as [5]

$$
\mathcal{P}_{n_{c}}(w)=c w^{n_{c}-1} e^{-n_{c} w},
$$

where $n_{c}$ is fixed for a given lattice and $c$ is a normalization constant. Note that these solutions have the same qualitative behavior as those found in our molecular dynamics simulations: for small weights $\mathcal{P}_{n_{c}}(w) \propto w^{n_{c}-1}$, and the probability for large weights decays exponentially.

The $q$ model is thus an effective minimal model for the weights $W$. It is clear that the product of $q_{i j}$ and $W_{i}$ has a natural interpretion as the vertical component of $\vec{F}_{i j}$. Since these interparticle forces are more fundamental than the weights, we investigate the statistics of the quantity $q W$ in Sec. VI A; this will shed light on the discrepancy for small forces between the $q$ model and experimental data. In the light of our finding that the contact geometry and in particular $n_{c}$ play a crucial role, the standard $q$ model is clearly limited since it fixes $n_{c}$. In Sec. VI B we therefore extend the $q$ model to have randomness in its connectivity (i.e., to allow for a range of $n_{c}$ 's) and find that, as expected, the $\mathcal{P}(w)$ can be manipulated by changes in the connectivity.

\section{A. Distribution of interparticle forces: $\boldsymbol{P}(q w)$}

A direct comparison of Eqs. (1) and (11) shows that the product $q_{i j} w_{i}$ has a natural interpretation as the vertical component of $\vec{f}_{i j}$. Since the interparticle forces are more important than the weights, it is interesting to investigate the statistical properties of the bond quantity $q w$. To obtain the distribution $P(q w)$, let us start with the transformation from $P(q w)$ to $\mathcal{P}_{n_{c}}(w)$ :

$$
\begin{aligned}
\mathcal{P}_{n_{c}}(w)= & \int_{0}^{\infty} d(q w)_{1} P\left((q w)_{1}\right) \cdots \int_{0}^{\infty} d(q w)_{n_{c}} P\left((q w)_{n_{c}}\right) \\
& \times \delta\left(w-\sum_{i=1}^{n_{c}}(q w)_{i}\right) .
\end{aligned}
$$

Here we assumed that the $(q w)_{i}$ are uncorrelated, which is valid for the uniform $q$ distribution [19]. For the corresponding Laplace transforms, denoted by $\widetilde{P}(s)$ and $\widetilde{\mathcal{P}}_{n_{c}}(s)$, respectively, this relation becomes

$$
\widetilde{\mathcal{P}}_{n_{c}}(s)=(\widetilde{P}(s))^{n_{c}} .
$$

Since the Laplace transform of Eq. (13) is of the form $1 /(1+s)^{n_{c}}$, the distribution of $q w$ reads

$$
\widetilde{P}(s)=\frac{1}{1+s} \Rightarrow P(q w)=e^{-q w} .
$$

We thus find (for the uniform $q$ distribution) that $P(q w)$ is a pure exponential, independent of the number of contacts, $n_{c}$. Again, this is very similar to the results for our Hertzian sphere packings: the distribution of "interparticle forces", $P(q w)$, is finite for small forces, whereas the distribution of weights depends on $n_{c}$ as given by Eq. (6). Moreover, this 


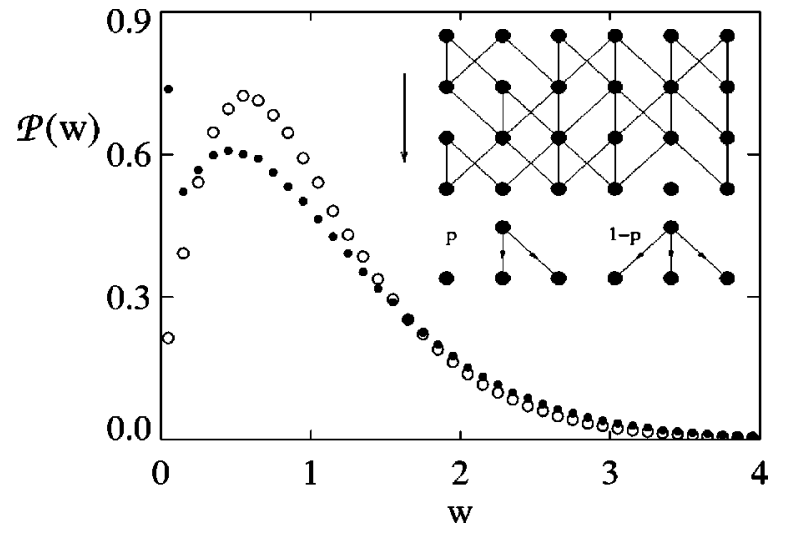

FIG. 10. The $q$ model with a random connectivity: with a probability $p$ we cut one of the three bonds (inset). We recover the bottom effect for $\mathcal{P}(w)$ in this model. In the bulk $p=0.3$ (open circles) and at the bottom $p=0.9$ (dots); this corresponds to $\left\{\rho_{0}, \rho_{1}, \rho_{2}, \rho_{3}\right\}=\{0.00,0.03,0.24,0.73\}$ and $\{0.03,0.19,0.44,0.34\}$, respectively.

resolves the discrepancy for small forces mentioned in the Introduction: the $q$ model predicts a vanishing probability densitity for small weights, but not for small forces.

\section{B. Including geometry effects}

From Sec. III, it is clear that the weight distribution $\mathcal{P}(w)$ in Hertzian sphere packings is very sensitive to the local packing geometry. Since the $q$ model is defined on a regular lattice, with fixed connectivity, it cannot capture the behavior of $\mathcal{P}(w)$ in disordered packings with fluctuating $n_{c}$. This extra degree of disorder can be included, for example, by "cutting" some of the bonds of the regular lattice. We illustrate this with the two-dimensional square lattice depicted in Fig. 10. For each site, the weight is transmitted downwards through either two or three bonds with probabilities $p$ and $1-p$, respectively; in the former case we randomly cut one of the available bonds and generate the two remaining $q_{i j}$ according to a uniform distribution satisfying Eq. (12). This generates particles with $n_{c}=0,1,2$, and 3 , since all bonds arriving at a site have a probability of $p / 3$ to be missing. For simplicity, we introduced the disorder in $n_{c}$ by means of one parameter $p$ only; as a consequence, we can only obtain a limited set of $\left\{\rho_{n_{c}}\right\}$.

With this model, we have tried to mimic the bulk-bottom behavior of $\mathcal{P}(w)$ that was observed in the amorphous packings [Fig. 5(a)]. In the bulk layers we took out bonds with probability $p=0.3$, and for the bottom layer we took $p=0.9$; the result is shown in Fig. 10. Indeed, the change in the fractions $\rho_{n_{c}}$ is sufficient to reproduce a transition of $\mathcal{P}(w)$ reminiscent of what has been observed in our Hertzian sphere packings [compare with Fig. 5(a)].

\section{Conclusions for the $q$ model}

Although it is known that the $q$ model does not properly describe the spatial structure of the force network [12], it remains a very instructive theoretical framework for the sta- tistics of force fluctuations. While in the standard case the disorder in the system is represented by the stochastic fractions $q_{i j}$ only, we have shown that when also the connectedness is chosen to be random, the model displays most features of realistic packings.

Let us conclude this section by mentioning that the idea to leave out some of the bonds of a regular lattice is not new [20]. In these studies, however, bonds were cut in a particular manner to build up long-ranged force correlations. We have shown that such long-ranged structures are not important for the behavior of $\mathcal{P}(w)$, since they only depend on the local packing geometry.

\section{TOP-DOWN RELAXATION OF FLUCTUATIONS}

So far, the discussion has been limited to situations well below the top surface of the packings. The data of the Hertzian sphere simulations were taken at least 15 layers below the top surface and the results of the $q$ model (presented in the previous section) all correspond to the limit of large depths. In this section we investigate the top-down relaxation of the force and weight distributions. At the top surface of the Hertzian sphere packings, there are only weight fluctuations due to grain polydispersity. The question we address is how fast the force and weight fluctuations build up towards a bulk distribution, as a function of depth.

These results can then be compared to the relaxation in the $q$ model. Interpreting the downward direction as time, this corresponds to transient behavior towards the "stationary" solutions given in Eqs. (13) and (16). This top-down relaxation of fluctuations forms an additional test to qualify various theoretical models, very much like the Green's function measuring the response to a localized load on the top surface [12]. In our case, we start from spatially (nearly) homogeneous conditions in the top layer and see how fluctuations build up.

\section{A. Top-down relaxation in Hertzian sphere packings}

A good way to quantify changes in $\mathcal{P}(w)$ and $P(f)$ is to study their second moments $\left\langle w^{2}\right\rangle$ and $\left\langle f^{2}\right\rangle$. For a distribution of zero width these second moments are unity, and they increase as the fluctuations become larger [21]. In Fig. 11 we show the second moments as a function of the height $h$, which is defined as the distance from the bottom boundary. Since the packings are strongly disordered, the precise location of the top surface will be slightly different for each realization; it turns out to be located around $h=46$.

Let us first consider the broadening of the weight distribution shown in Fig. 11(a). As already mentioned above, the weight fluctuations at the top surface are entirely due to the polydispersity of the grains. Using a flat distribution between $0.4<r<0.6$ this corresponds to $\left\langle w^{2}\right\rangle \approx 1.11$, which is consistent with our simulation data. The second moment approaches its bulk value already at a depth of approximately ten particle diameters. The figure also shows the sharp transition of $\mathcal{P}(w)$ at the bottom boundary. The second moments of $P(f)$ are shown in Fig. 11(b). One again observes a relaxation over approximately ten layers, towards a bulk value; 

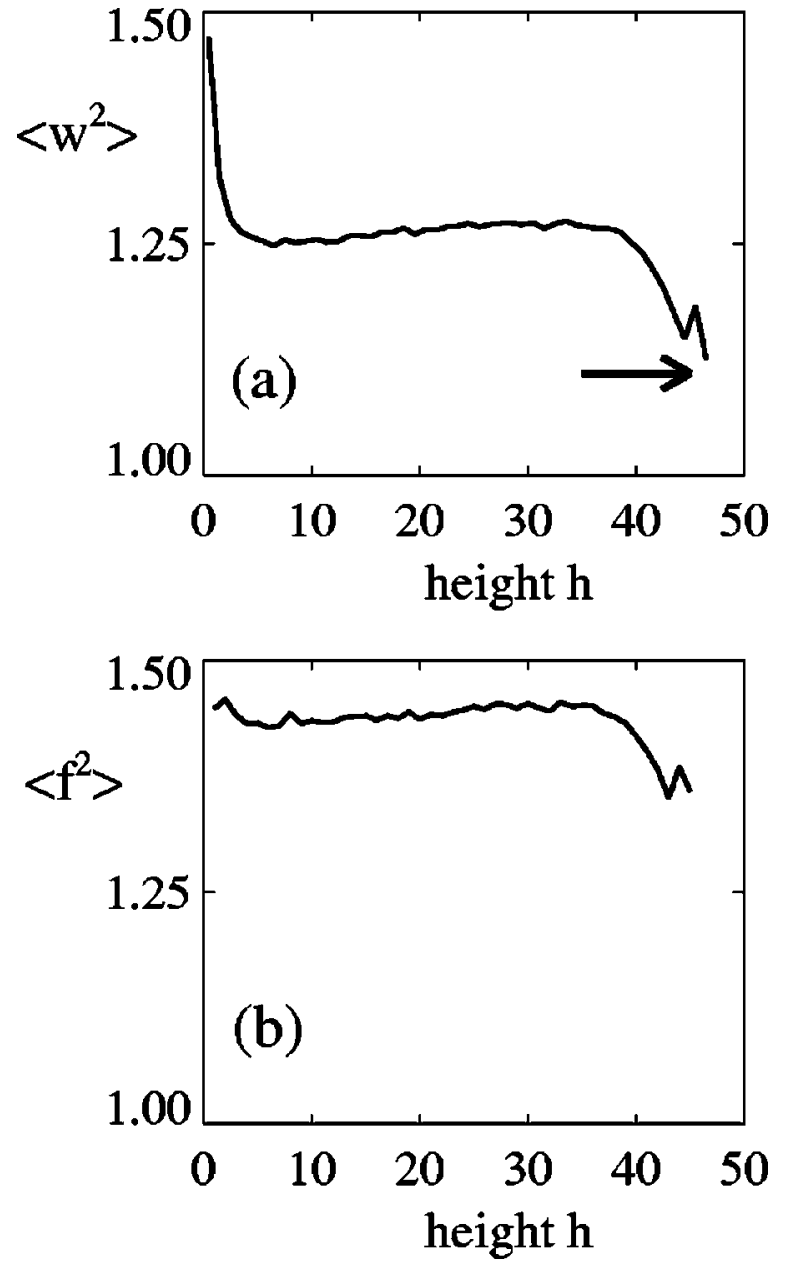

FIG. 11. The second moments (a) $\left\langle w^{2}\right\rangle$ and (b) $\left\langle f^{2}\right\rangle$ as a function of height $h$ in simulations of Hertzian sphere packings. The arrow indicates the location of the top surface, around $h=46$. For both the forces and the weights one finds a fast top-down relaxation of the moments.

$P(f)$ does not change significantly near the bottom boundary. Note that both the force and weight distributions become slightly narrower as the depth increases below heigths of the order of 30. This may be attributed to an increase in particle deformations [15].

We thus find that the typical length scale for force and weight fluctuations to saturate is approximately ten particle diameters. This provides another important criterion to distinguish between different theoretical models.

\section{B. Top-down relaxation in the $q$ model}

The top-down relaxation is well understood for the $q$ model without the so-called injection term-i.e., $m g=0$ in Eq. (11) $[19,22]$. Before extending these results to the $q$ model with injection [23], we briefly recapitulate the results of the $q$ model without the injection term $m g$. This version of the model can be interpreted as a packing of weightless particles, supporting a homogeneously applied force. To distinguish between the $q$ model without injection from the model with injection, we denote the weight distributions at depth $t$ by $\mathcal{R}^{(t)}(w)$ (without injection) and by $\mathcal{P}^{(t)}(w)$ (with injection).

For the uniform $q$ distribution, it has been shown that [19]

$$
\mathcal{R}^{(t)}(w) \simeq \mathcal{P}(w)+\left(\frac{1}{\sqrt{t}}\right)^{d-1} \mathcal{F}(w) \text { for } \mathrm{t} \rightarrow \infty,
$$

where $d$ is the dimensionality of the packing. The stationary solution $\mathcal{P}(w)$ is given by Eq. (13) and $\mathcal{F}(w)$ is the shape of a typical deviation. It is clear that all second- and higherorder moments $\left\langle w^{k}\right\rangle$ approach their asymptotic values according to the same power law. This slow relaxation towards $\mathcal{P}(w)$ is caused by the diffusion of correlations, which takes place in $(d-1)$-dimensional correlation space [24].

Let us now investigate how the injection term $m g$ affects the top-down relaxation. We first note that the recursive relation for the weights, Eq. (11), is a linear equation. The $q$ model with injection can therefore be interpreted as a superposition of $q$ models without injection, with differently positioned initial layers. Although it is not a priori clear how this superposition property is reflected in the weight distributions $\mathcal{R}^{(t)}(w)$ (with injection) and $\mathcal{R}^{(t)}(w)$ (without injection), we propose the following approximate mapping:

$$
\mathcal{P}^{(t)}(w)=\frac{1}{t+1} \sum_{t^{\prime}=0}^{t} \mathcal{R}^{\left(t^{\prime}\right)}(w) .
$$

If we combine this with the exact result of Eq. (17), we obtain the following relaxation as $t \rightarrow \infty$ :

$$
\begin{aligned}
\mathcal{P}^{(t)}(w)-\mathcal{P}(w) & \propto \mathcal{F}(w) \frac{1}{t+1} \sum_{t^{\prime} \neq 0}^{t}\left(\frac{1}{\sqrt{t^{\prime}}}\right)^{d-1} \\
& \propto \mathcal{F}(w)\left\{\begin{array}{cc}
\frac{1}{\sqrt{t}}, & d=2, \\
\frac{\log (t)}{t}, & d=3, \\
\frac{1}{t}, & d \geqslant 4 .
\end{array}\right.
\end{aligned}
$$

This relaxation behavior is indeed observed in our numerical simulations with $d=2$ and $d=3$, using a uniform $q$ distribution. In Fig. 12, we show the results for an fcc packing $(d$ $=3$ ). We plot $t\left|\left\langle w^{2}\right\rangle^{(t)}-4 / 3\right|$ as function of depth $t$, where $\left\langle w^{2}\right\rangle^{(\infty)}=4 / 3$. The climbing straight line on the lin-log plot confirms the remarkable $\log (t) / t$ relaxation. This result is also implicit in Ref. [23]. We also plot the same data for the $q$ model without injection; this curve becomes flat, in agreement with Eq. (17).

Although the mapping of Eq. (18) is definitely not exact, it apparently captures the main physics of the relaxation process. This can be understood as follows. There are two slow processes involved: (i) the increasing number of layers reduces the contribution of each layer of "injected" weights effectively as $1 / t$; (ii) each layer of injected weights relaxes as $(1 / \sqrt{t})^{(d-1)}$ individually. Naturally, the total relaxation is dominated by the slower of these two processes. In the special case of $d=3$ both powers are $1 / t$, leading to a logarith- 


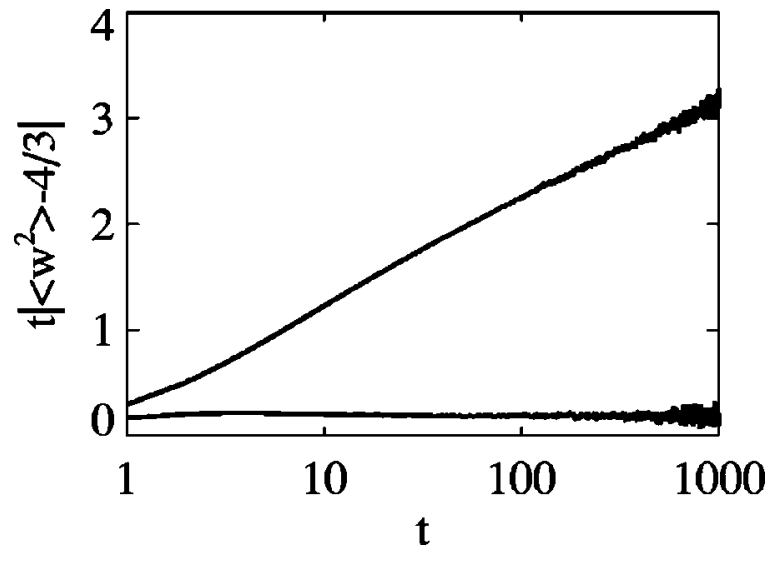

FIG. 12. Relaxation of the second moments with injection (climbing line) and without injection (flat line) towards their asymptotic values $4 / 3$ in the 3D $q$ model. Since we plot $t \mid\left\langle w^{2}\right\rangle^{(t)}$ $-4 / 3$ | along the vertical axis, the climbing straight line confirms the $\log (t) / t$ relaxation for the $q$ model with injection. Without injection the relaxation is simply $1 / t$.

mic correction. Finally note that since the downward $q$ values are statistically independent from the weights, the "force" fluctuations simply follow from $\left\langle(q w)^{2}\right\rangle=\left\langle q^{2}\right\rangle\left\langle w^{2}\right\rangle$ and thus display the same relaxation as the weights fluctuations.

\section{Conclusions concerning top-down relaxation}

We have studied the top-down relaxation of the second moments $\left\langle w^{2}\right\rangle$ and $\left\langle f^{2}\right\rangle$, which quantifies how "fast" the weight and force distributions approach their bulk shapes. The $q$ model predicts a power-law relaxation with a logarithmic correction for 3D packings, Eq. (19). However, we find no evidence for such a slow relaxation in our simulations of Hertzian spheres, which indicate that a bulk distribution is reached after approximately ten layers of particles (Fig. 11). In the $q$ model with injection, for example, the second moment after ten layers still differs around $20 \%$ from its asymptotic value.

Let us provide two possible explanations why the $q$ model fails to describe this relaxation process. A first problem of the model is that it assumes some fixed $q$ distribution $\eta(q)$ : we have seen that the $q$ 's can in principle be derived from the forces as $q_{i j}=\left(\vec{F}_{i j}\right)_{z} / W_{i}$, so a relaxation in $P(f)$ and $\mathcal{P}(w)$ should result in a relaxation of $\eta(q)$ itself. This clearly shows the difficulty of encoding the force behavior into a stochastic variable $q$ in a self-consistent manner. Another problem of the model is that it assumes a top-down propagation of forces. The up-down symmetry is therefore broken explicitly in the $q$ model, whereas in our Hertzian sphere packings we find only a very weak symmetry breaking. In principle, force networks are defined by the equations of mechanical equilibrium, which generically are underdetermined [25,26] and hence cannot be solved by an iterative (top-down) procedure. Instead, one has to solve this set of coupled equations "simultaneously" for all particles in the system, and except for the (small) $m g$ term, there is a natural up-down symmetry in this system. The absence of this up-down symmetry in the $q$ model could of course strongly affect the top-down relaxation.

\section{DISCUSSION}

We have argued that in order to understand the statistics of forces in granular packings, it is crucial to distinguish between interparticle forces and weights. We have found in our simulations that the force distribution $P(f)$ is very robust, in the sense that its shape does not depend on details of packing geometry. The weight distribution $\mathcal{P}(w)$, on the other hand, is very sensitive to the local packing geometry. We have demonstrated that a decomposition according to the number of contacts that press on a particle from above, $n_{c}$, is sufficient to understand this geometry dependence. Reinterpreting experiments on strongly deformed rubber particles [11] within this framework, we find strong evidence that $P(f)$ essentially remains unaffected even by very large particle deformations. To further test our framework experimentally, one can manipulate the number of contacts at the boundary by placing a layer of relatively small or large beads at the bottom. For small beads, the fractions $\rho_{0}$ and $\rho_{1}$ will be enhanced, leading to a large $\mathcal{P}(w)$ for small $w$ and a slow exponential decay for large $w$. Relatively large bottom beads should lead to a $\mathcal{P}(w)$ that is strongly peaked.

The present work provokes a number of questions. First, we observe that most of our simulation results, like the shapes of $P^{\prime}\left(f_{z}\right)$ and $\mathcal{P}(w)$, can to a large extent be understood in terms of local packing geometry only. This suggests that at least for the "one-point" force, weight, and angle probability distributions, long-range correlations are not dominant. We therefore question whether the behavior of $P(f)$ observed at the jamming transition [6,7] reflects a longrange structural change of the force network. In particular, we expect that the role of "force chains" can only be understood from two- or more-point correlation functions, and not from $P(f)$ only.

A related problem is that the $q$ model fails to describe problems that involve the spatial structure of the force network. Although the model is able to capture many features of force and weight statistics (Sec. VI), it does not produce the top-down relaxation of $\mathcal{P}(w)$ that is observed in the more realistic Hertzian packings. Alongside the incorrect prediction of the response function [12], this indicates that spatial dependence is not correctly incorporated within the $q$ model. This may be due to the fact that, in general, recursive models do not acknowledge the structure of the equations describing mechanical equilibrium. These equations are typically underdetermined [25] and cannot be solved in a recursive manner. In a recent paper [26], we therefore propose a different theoretical approach, in which we start from the equations of mechanical stability and exploit the undetermined degrees of freedom.

Another important issue for future study is clearly the role of friction and dimensionality. Our numerical study has been done in two dimensions with frictionless spheres; however, recent studies indicate [15] that the coordination number for $3 \mathrm{D}$ packings with friction is similar to those of $2 \mathrm{D}$ friction- 
less packings. Qualitatively, the picture we have advanced is therefore expected to capture the realistic case of three dimensions with friction, because our phase-space arguments are independent of dimension.

\section{ACKNOWLEDGMENTS}

We are very grateful to Nathan Mueggenburg and Heinrich Jaeger for providing some of their experimental data and for the open exchange of ideas. We also thank Martin Howard, Hans van Leeuwen, and Carlo Beenakker for numerous illuminating discussions. J.H.S. and E.S. gratefully acknowledge support from the physics foundation FOM and $\mathrm{MvH}$ support from the science foundation NWO through a VIDI grant.

\section{APPENDIX A: LOGARITHMIC DIVERGENCE OF $P^{\prime}\left(f_{z}\right)$}

In Sec. II C, we encounter the following integral:

$$
\begin{aligned}
P^{\prime}\left(f_{z}\right) & =\int_{0}^{\pi} d \varphi \frac{1}{\pi} \int_{0}^{\infty} d f P(f) \delta\left(f_{z}-f \sin (\varphi)\right) \\
& =\int_{0}^{\infty} d f P(f) \int_{0}^{\pi / 2} d \varphi \frac{2}{\pi} \frac{1}{f} \delta\left(\frac{f_{z}}{f}-\sin (\varphi)\right) \\
& =\frac{2}{\pi} \int_{f_{z}}^{\infty} d f \frac{1}{\sqrt{f^{2}-f_{z}^{2}}} P(f) .
\end{aligned}
$$

The function $P(f)$ represents the probability density function of $f=|\vec{f}|$, which we can assume to be regular on the entire interval (see Fig. 2). The behavior for small $f_{z}$ is not trivial, since the integrand diverges at the lower bound of the integration interval. For each nonzero $f_{z}$ this does not lead to a singularity, since

$$
P^{\prime}\left(f_{z}\right)=\frac{2}{\pi} \int_{f_{z}}^{\infty} \frac{d f}{f_{z}} \frac{P(f)}{\sqrt{\left(f / f_{z}\right)^{2}-1}}=\frac{2}{\pi} \int_{1}^{\infty} d u \frac{P\left(u f_{z}\right)}{\sqrt{u^{2}-1}} .
$$

The integral over $1 / \sqrt{u^{2}-1}$ is convergent for $u \rightarrow 1$ and the function $P\left(u f_{z}\right)$ falls fast enough as $\left(u f_{z}\right) \rightarrow \infty$. For $f_{z}=0$, however, the integral diverges as $u \rightarrow \infty$. To obtain the asymptotic behavior we rewrite the integral as

$$
P^{\prime}\left(f_{z}\right)=\frac{2}{\pi} \int_{1}^{\infty} d u \frac{P\left(u f_{z}\right)}{u}+\frac{2}{\pi} \int_{1}^{\infty} d u P\left(u f_{z}\right)\left(\frac{1}{\sqrt{u^{2}-1}}-\frac{1}{u}\right) .
$$

The second term is convergent since the term between brackets behaves as $1 / u^{3}$ as in the limit $u \rightarrow \infty$. We thus find that

$$
P^{\prime}\left(f_{z} \rightarrow 0\right) \simeq \frac{2}{\pi} \int_{f_{z}}^{\infty} d f \frac{P(f)}{f}+\mathcal{O}(1) \simeq-\frac{2}{\pi} P(0) \ln \left(f_{z}\right)+\mathcal{O}(1) \text {. }
$$

\section{APPENDIX B: RELATION BETWEEN TAILS OF $\boldsymbol{P}^{\prime}\left(f_{z}\right)$ AND $\mathcal{P}_{n_{c}}(w)$}

In this appendix we derive the large weight behavior of from the tail of $\mathcal{P}_{n_{c}}(w)$ from the tail of $P^{\prime}\left(F_{z}\right)$, assuming that the various $\left(\vec{F}_{i}\right)_{z}$ in Eq. (5) are uncorrelated. We consider decays both faster and slower than exponential, of the form

$$
P^{\prime}\left(F_{z}\right) \propto \exp \left(-\alpha F_{z}^{\beta} /\left\langle F_{z}\right\rangle^{\beta}\right) \text { for } F_{z} \rightarrow \infty .
$$

We show that, after rescaling $\langle w\rangle$ to unity, this leads to

$$
\mathcal{P}_{n_{c}}(w) \propto e^{-\gamma w^{\beta}},
$$

with

$$
\gamma= \begin{cases}\alpha n_{c}, & \beta \geqslant 1, \\ \alpha n_{c}^{\beta}, & \beta \leqslant 1 .\end{cases}
$$

This means that the tail of the weight distribution is of the same nature as that of the forces, but with a different prefactor $\gamma$. The tails get steeper for increasing $n_{c}$, since the reduced probability for small $w$ (due to a lack of phase space) must be compensated to keep $\langle w\rangle=1$.

The above results are obtained as follows. Rescaling all forces in Eq. (5) as $x_{i}=\left(F_{z}\right)_{i} / W$, one obtains the probability for large weights:

$$
\begin{aligned}
\mathcal{P}_{n_{c}}(W) \propto & W^{n_{c}-1} \int_{\mathcal{S}} d x_{1} \cdots d x_{n_{c}} \\
& \times \exp \left(-\frac{\alpha}{\left\langle F_{z}\right\rangle^{\beta}} W^{\beta}\left(x_{1}^{\beta}+\cdots+x_{n_{c}}^{\beta}\right)\right),
\end{aligned}
$$

where $\mathcal{S}$ denotes the hyperplane $1-\sum_{i} x_{i}$ with all $x_{i} \geqslant 0$.

For $\beta>1$, the probability density on $\mathcal{S}$ has a maximum at $x_{i}=1 / n_{c}$, which becomes sharply peaked for increasing $W$. Physically, this means that the dominant contribution for large weights will come from all $F_{z}$ being equal-namely, $W / n_{c}$. Approximating the integrand by a Gaussian around its maximum value, we find that the "width" decreases as a power of $W$ only-namely, $1 / W^{\left(n_{c}-1\right) \beta / 2}$. Hence the leading behavior for large $W$ is given by the maximum value of the integrand-i.e., $\exp \left[-\left(\alpha /\left\langle F_{z}\right\rangle^{\beta}\right) W^{\beta} /\left(n_{c}\right)^{\beta-1}\right]$.

For $\beta<1$, the probability density has a minimum at $x_{i}$ $=1 / n_{c}$, and the dominant contribution now comes from $x_{i}$ $=1$ and $x_{j \neq i}=0$. This means that typically only one of the forces accounts for the whole weight. The part of the integral around $x_{i}=1$ can be approximated by

$$
\exp \left(-\frac{\alpha}{\left\langle F_{z}\right\rangle^{\beta}} W^{\beta}\right) \int_{\mathcal{S}_{\epsilon}} d x_{1} \cdots d x_{n} \exp \left(-\frac{\alpha}{\left\langle F_{z}\right\rangle^{\beta}} W^{\beta} \sum_{j \neq i} x_{j}^{\beta}\right),
$$

where $\mathcal{S}_{\epsilon}$ denotes the part of $\mathcal{S}$ for which $1-x_{i} \leqslant \epsilon$. This approximation becomes exact for $W \rightarrow \infty$ as long as $W^{\beta} \epsilon$ $\ll 1$; we take $\epsilon=1 / W^{1-\delta}$ with $0<\delta<1-\beta$. Working out the integration over $\mathcal{S}_{\epsilon}$, one finds

$$
\frac{\exp \left(-\frac{\alpha}{\left\langle F_{z}\right\rangle^{\beta}} W^{\beta}\right)}{W^{n_{c}-1}}\left[\int_{0}^{\infty} d y \exp \left(-\frac{\alpha}{\left\langle F_{z}\right\rangle^{\beta}} y^{\beta}\right)\right]^{n_{c}-1},
$$

as $W \rightarrow \infty$. The part of the integral outside the areas $\mathcal{S}_{\epsilon}$ is 
smaller than $W^{n_{c}-1} \exp \left[-\left(\alpha /\left\langle F_{z}\right\rangle^{\beta}\right) W^{\beta}\left(1+W^{\delta}\right)\right]$ and can thus be neglected. So also for $\beta<1$, the leading behavior for large $W$ is simply given by the maximum value-i.e., $\exp \left[-\left(\alpha /\left\langle F_{z}\right\rangle^{\beta}\right) W^{\beta}\right]$.
As mentioned in Sec. III, the $\mathcal{P}_{n}(W)$ obtained by Eq. (5) are not properly normalized, since $\langle W\rangle=\left\langle f_{z}\right\rangle n_{c}$. If we rescale the average weight to unity, we obtain the results of Eqs. (B2) and (B3).
[1] H. M. Jaeger, S. R. Nagel, and R. P. Behringer, Rev. Mod. Phys. 68, 1259 (1996); P. G. de Gennes, ibid. 71, 374 (1999).

[2] D. M. Mueth, H. M. Jaeger, and S. R. Nagel, Phys. Rev. E 57, 3164 (1998); D. L. Blair, N. W. Mueggenburg, A. H. Marshall, H. M. Jaeger, and S. R. Nagel, ibid. 63, 041304 (2001).

[3] G. Løvoll, K. J. Måløy, and E. G., Flekkøy, Phys. Rev. E 60, 5872 (1999).

[4] F. Radjai, M. Jean, J. J. Moreau, and S. Roux, Phys. Rev. Lett. 77, 274 (1996); S. Luding, Phys. Rev. E 55, 4720 (1997); F. Radjai, D. E. Wolf, M. Jean, and J. J. Moreau, Phys. Rev. Lett. 80, 61 (1998); A. V. Tkachenko and T. A. Witten, Phys. Rev. E 62, 2510 (2000); S. J. Antony, ibid. 63, 011302 (2000); C. S. O'Hern, S. A. Langer, A. J. Liu, and S. R. Nagel, Phys. Rev. Lett. 88, 075507 (2002).

[5] C. Liu, S. R. Nagel, D. A. Schecter, S. N. Coppersmith, S. Majumdar, O. Narayan, and T. A. Witten, Science 269, 513 (1995); S. N. Coppersmith, C. Liu, S. Majumdar, O. Narayan, and T. A. Witten, Phys. Rev. E 53, 4673 (1996).

[6] C. S. O’Hern, S. A. Langer, A. J. Liu, and S. R. Nagel, Phys. Rev. Lett. 86, 111 (2001).

[7] L. E. Silbert, D. Ertas, G. S. Grest, T. C. Halsey, and D. Levine, Phys. Rev. E 65, 051307 (2002).

[8] J. H. Snoeijer, M. van Hecke, E. Somfai, and W. van Saarloos, Phys. Rev. E 67, 030302(R) (2003).

[9] D. Howell, R. P. Behringer, and C. Veje, Phys. Rev. Lett. 82, 5241 (1999).

[10] J. Brujic, S. F. Edwards, D. V. Grinev, I. Hopkinson, D. Brujic, and H. A. Makse, Faraday Discuss. 123, 207 (2003).

[11] J. M. Erikson, N. W. Mueggenburg, H. M. Jaeger, and S. R. Nagel, Phys. Rev. E 66, 040301 (2002).

[12] G. Reydellet and E. Clement, Phys. Rev. Lett. 86, 3308 (2001); J. Geng, D. Howell, E. Longhi, R. P. Behringer, G. Reydellet, L. Vanel, E. Clement, and S. Luding, ibid. 87, 035506 (2001); N. W. Mueggenburg, H. M. Jaeger, and S. R. Nagel, Phys. Rev. E 66, 031304 (2002); D. A. Head, A. V. Tkachenko, and T. A. Witten, Eur. Phys. J. E 6, 99 (2001); C. Goldenberg and I. Goldhirsch, Phys. Rev. Lett. 89, 084302 (2002).

[13] K. L. Johnson, Contact Mechanics (Cambridge University Press Cambridge, England, 1985).

[14] There is a slight difference between the packings of "soft" particles (deformation $0.1 \%$ ) and "hard" particles (deformation $0.1 \%$ ). The average coordination number of the hard particles is smaller than that of the soft particles, leading to a significant number of rattlers-i.e., particles that feel no force from above. Since the forces carrying these rattlers are of the order $m g \ll\langle F\rangle$, they show up as $\delta$ peaks at zero force (see also Sec. II B); besides these $\delta$ peaks, $P(f)$ in both systems are virtually indistinguishable.

[15] H. A. Makse, D. L. Johnson, and L. M. Schwartz, Phys. Rev. Lett. 84, 4160 (2000).

[16] When determining the value of $n_{c}$, we explicitly exclude the intralayer bottom contacts; for the strongly polydisperse packings, these correspond to angles that deviate less than $13^{\circ}$ from the horizontal. For our estimates of the bulk $\rho_{n_{c}}$ 's we do not exclude any forces; i.e., the "cutoff angle" is strictly $0^{\circ}$. Since the bulk is isotropic, a cutoff angle of $13^{\circ}$ leads to a change of the order of $15 \%$, far too little to explain the change of $\rho_{1}$ by a factor of more than 4 . For simplicity we therefore have kept a "cutoff angle" of $0^{\circ}$ in the bulk.

[17] To determine the precise value of $\mathcal{P}_{1}(0)$ is a very subtle problem. Since the total weight distribtution $\mathcal{P}(w)$ is normalized such that $\langle w\rangle=1$, the average weight of particles with $n_{c}=1$ will be smaller than unity and $\mathcal{P}_{1}(w)$ should be rescaled with a factor $\left\langle n_{c}\right\rangle$. Incorporating this in a self-consistent manner, we obtained very similar $\left\langle n_{c}\right\rangle$ as those presented in Table 1, where we took $\mathcal{P}_{1}(0)=0.5$ for simplicity.

[18] L. E. Silbert, G. S. Grest, and J. W. Landry, Phys. Rev. E 66, 061303 (2002).

[19] J. H. Snoeijer and J. M. J. van Leeuwen, Phys. Rev. E 65, 051306 (2002); J. Stat. Phys. 109, 449 (2002).

[20] P. Claudin and J.-P. Bouchaud, Phys. Rev. Lett. 78, 231 (1997); M. Nicodemi, ibid. 80, 1340 (1998).

[21] The second moments are very sensitive to small changes in the distributions and hence form a good representation of the evolution of the entire distributions $\mathcal{P}(w)$ and $\mathcal{P}(f)$ as a function of depth. To see what the corresponding distributions look like, we refer to Figs. 2 and 5(a).

[22] M. Lewandowska, H. Mathur, and Y.-K. Yu, Phys. Rev. E 64, 026107 (2001)

[23] R. Rajesh and S. N. Majumdar, Phys. Rev. E 62, 3186 (2000).

[24] This diffusion in correlation space should not be confused with the diffusion in real space due to a localized force on the top surface. In our case we have homogeneous initial conditions as all weights in the top layer are equal.

[25] L. E. Silbert, D. Ertas, G. S. Grest, T. C. Halsey, and D. Levine, Phys. Rev. E 65, 031304 (2002).

[26] J. H. Snoeijer, T. J. H. Vlugt, M. van Hecke, and W. van Saarloos, Phys. Rev. Lett. 92, 054302 (2004). 